\title{
Modelo Colaborativo y Ubicuo para apoyar los procesos de enseñanza-aprendizaje a nivel Iberoamericano
}

\section{Collaborative and Ubiquitous Model to Support Teaching and Learning Processes in Iberoamerica}

\author{
Mayela Coto \\ Universidad Nacional. Costa Rica. \\ mcoto@una.cr \\ César A. Collazos \\ Universidad del Cauca, Colombia. \\ ccollazo@unicauca.edu.co \\ Sonia Mora Rivera \\ Universidad Nacional. Costa Rica. \\ smora@una.cr
}

\begin{abstract}
Resumen
Los nuevos esquemas económicos plantean retos para los profesionales, debido a que se hace necesario la adquisición de mejores niveles de conocimiento, la continua actualización de la información, la adquisición de habilidades tecnológicas, la capacidad de síntesis y el pensamiento crítico. Esto implica la necesidad de que los sistemas educativos actuales evolucionen, con el objetivo de seguir preparando a las personas, contando por supuesto con las ventajas que ofrecen las nuevas tecnologías de la información y la comunicación. El u-learning o aprendizaje ubicuo, es un nuevo modelo de aprendizaje que describe un conjunto de actividades formativas, apoyadas en el uso de tecnología, que pueden ser accesibles en cualquier lugar y de cualquier forma. Este artículo presenta un modelo de enseñanza-aprendizaje colaborativo y ubicuo (u-CSCL) a partir del uso de herramientas tecnológicas actuales, utilizando diferentes dispositivos de interacción, permitiendo no sólo aprender continuamente sino adquirir habilidades comunicativas, de cooperación y trabajo grupal. Su desarrollo ha sido liderado en conjunto por un grupo de investigadores de universidades a nivel Iberoamericano. Además se presenta el diseño de una plataforma tecnológica que soporta el modelo y se hace una valoración inicial de la misma, con un grupo reducido de participantes, que permitió obtener datos preliminares conducentes a la mejora de la plataforma, pero también ratificaron que la herramienta propuesta permite y facilita a los docentes la creación de cursos de forma colaborativa y ubicua, y a los estudiantes les brinda la posibilidad de participar en las actividades colaborativas propuestas, propiciando así el desarrollo de conocimiento conjunto. En una segunda fase se proponer seguir validando el entorno, para obtener mayores resultados y más precisos.
\end{abstract}

\section{Palabras clave}

Aprendizaje colaborativo, aprendizaje ubicuo, CSCL.

\footnotetext{
Abstract

The new professionals require higher levels of knowledge, continuous updating of information, and acquisition of technological skills, synthesis and critical thinking in order to adapt to the new economic models. This implies the need for current educational systems to evolve in order to continue preparing people, considering the advantages offered by new
} 
information and communications technology. The u-learning or ubiquitous learning is a new learning model available in different channels simultaneously and in a fully distributed environment, describing a series of training activities, supported by the use of technology, which can be accessed anywhere, anyway. This article describes a model of ubiquitous and collaborative teaching and learning using different interaction devices, allowing not only to learn but to acquire communication, cooperation, and teamwork skills continuously. This model was led by a team of Iberoamerican researchers. Besides, a technological platform design is presented in order to support the model proposed, and a preliminary validation is performed using a reduced group of participants, obtaining interesting preliminary results, where the software tool was considered adequate for teachers to create courses in a collaborative and ubiquitous manner, and for students to participate in a better way. A validation with a bigger group of students and teachers is proposed for a further work.

Key words

Collaborative learning, ubiquitous learning, CSCL.

\section{Introducción}

Cada día crece la tendencia hacia el trabajo en forma colaborativa para alcanzar metas comunes. Cuando se integran aspectos colaborativos a un proceso determinado se busca una mejora en la comunicación, y una mayor participación y compromiso entre los integrantes de un grupo, lo que redunda en una mayor productividad y una mejor calidad del producto final. El proceso de crear, en forma colaborativa recursos de aprendizaje forma parte de esta tendencia colaborativa. Este artículo presenta las bases teóricas de un modelo colaborativo basado en la ingeniería de la colaboración (IC) desarrollado de manera colaborativa por un equipo de investigadores a nivel Iberoamericano y su aplicación en un ambiente de enseñanza-aprendizaje ubicuo y colaborativo. El trabajo se realiza en el marco de la red CYTED u-CSCL que está conformada por 80 investigadores de 12 universidades pertenecientes a 7 países iberoamericanos, la experiencia en sí misma involucra el trabajo colaborativo. En esta red participan las siguientes Universidades: Universidad del Cauca- Colombia; Universidad Nacional de Manizales-Colombia; Universidad Unicomfacauca-Colombia; Universidad Nacional de Costa Rica; Universidad Tecnológica de Panamá; Universidad Nacional de San Juan-Argentina; Universidade Federal do Rio Grande do Sul- Brasil; Pontificia Universidad Católica de Valparaíso-Chile; Universidad de la Laguna, España; Universidad de Granada-España; Universidad de Lleida-España; Universidad de Castilla la Mancha-España y la Universidade Portucalense-Portugal.

Dicho modelo utiliza como base la "Metodología para el Desarrollo de Procesos Colaborativos" de Kolfschoten y Vreede (2007). El objetivo es que dicha metodología pueda ser aplicada a la creación colaborativa de recursos de aprendizaje en la que participen varias personas que pueden o no estar distribuidas geográficamente y que puedan pertenecer a diferentes áreas de conocimiento (Solano \& Collazos, 2013).

La estructura del artículo es la siguiente, primeramente se presentan, de forma breve, los referentes teóricos que sustentan el modelo, luego se describe la metodología en la cual se basa el diseño del mismo, posteriormente se explica y conceptualiza el modelo y el diseño de la plataforma que lo soporta y se hace una valoración inicial de la misma, finalmente se enuncia el trabajo a futuro. 


\section{Referentes teóricos}

A continuación se presentan los principales referentes teóricos que sustentan el UCSCL: modelo colaborativo y ubicuo para apoyar los procesos de enseñanzaaprendizaje, de manera breve se hace referencia a los conceptos más importantes que soportan el mismo.

\subsection{Aprendizaje colaborativo}

De acuerdo a Badia (2005), en la sociedad actual se está imponiendo una perspectiva más social e interdependiente de cada uno de los miembros de una sociedad, en la que se requiere la necesidad ineludible de colaboración con los otros, y donde cada vez tienen menos cabida las personas que actúan como un ser aislado e independiente.

El término de aprendizaje colaborativo se refiere a una estrategia de enseñanzaaprendizaje en la que los participantes tienen que colaborar con otros para cumplir con un objetivo de aprendizaje y alcanzar una tarea determinada. Esto conlleva la necesidad de un compromiso mutuo establecido entre los participantes y de un esfuerzo coordinado para dar respuesta a la tarea asignada (Muhlenbrock, 1999). Así, esta manera de aprender se aleja de metodologías individualistas o competitivas que no requieren la interacción con otros estudiantes (Johnson \& Johnson, 1975).

Johnson y otros (1992) destacan las siguientes características del aprendizaje colaborativo: (1) interdependencia positiva: al perseguir un objetivo común los miembros del grupo están motivados a ayudarse mutuamente; (2) fomento de la interacción: los miembros de un grupo deben interactuar unos con otros para resolver el problema eficientemente, mediante los aportes individuales; (3) responsabilidad individual: cada uno de los miembros del grupo asume su responsabilidad para contribuir al aprendizaje de todos; (4) desarrollo de competencias: trabajando conjuntamente los estudiantes desarrollan una serie de competencias académicas propias de la problemática a resolver, así como competencias interpersonales, como trabajo en equipo, liderazgo, capacidad de negociación, criticidad, etc.; (5) valoración de los miembros del grupo: mantener una buena relación de colaboración con los demás y estar dispuesto a dar y recibir comentarios y críticas constructivas sobre la contribución individual y del grupo.

Todas son características que hacen del aprendizaje colaborativo una forma de aprendizaje deseable. De acuerdo a Barkley, Cross y Major (2005), los dos pilares principales del aprendizaje colaborativo son la interdependencia positiva y la responsabilidad individual de cada estudiante. Adicionalmente, la clave para entender el aprendizaje colaborativo es reconocer las relaciones que se establecen entre la situación que se plantea, las interacciones que emergen y en consecuencia, los procesos y efectos que se generan en ella (Dillenbourg, 1999)

Cuando hay aprendizaje colaborativo cada uno de los miembros del grupo debe estar comprometido con la meta del grupo, y ser consciente de que su contribución al grupo no es competitiva sino que forma parte de una interdependencia positiva donde el logro de la meta conjunta es más importante que las contribuciones individuales (Scagnoli \& Stephens, 2005). 
Calzadilla (2002) indica que el aprendizaje colaborativo facilita la desaparición de los estudiantes como observadores pasivos y receptores repetitivos, generando espacios para la confrontación de múltiples perspectivas y la negociación en un proceso de aprendizaje que conduce al desarrollo integral de los participantes, capacidades deseables en los nuevos profesionales. De esta manera el aprendizaje colaborativo genera espacios con múltiples posibilidades y oportunidades de intercambio, lo que fomenta el desarrollo de habilidades individuales y grupales a partir de la negociación entre los estudiantes, potencia el crecimiento del grupo y una mejor transferencia de conocimiento que el aprendizaje individual (Kirschner, Paas, \& Kirschner, 2009; Rubia, Jorri, \& Anguita, 2009).

De esta manera, el aprendizaje colaborativo no es un mecanismo simple, ya que si se habla acerca de "aprender de la colaboración", también debe hablarse de "aprender por el hecho de estar solo". Los sistemas cognitivos de los individuos no aprenden porque ellos sean individuales, sino porque ejecutan algunas actividades (leer, predecir, etc.) que conllevan algunos mecanismos de aprendizaje (inducción, predicción, compilación, etc.). Similarmente, los pares no aprenden porque sean dos, sino porque ellos ejecutan algunas actividades que conllevan mecanismos de aprendizaje específicos. Esto incluye las actividades y/o mecanismos ejecutadas individualmente, pero además, la interacción entre sujetos genera actividades adicionales (explicación, regulaciones mutuas, etc.). El área de aprendizaje colaborativo, precisamente se refiere a éstas actividades y mecanismos. Estos pueden ocurrir con mayor frecuencia en un aprendizaje colaborativo que en condiciones individuales. Sin embargo, no hay garantía alguna de que estos mecanismos ocurran en cualquier interacción colaborativa. Por otra parte, ellos no ocurren solamente durante la colaboración. A cierto nivel de descripción- al menos a un nivel neuronal- los mecanismos potencialmente envueltos en un aprendizaje colaborativo son los mismos que aquellos potencialmente envueltos en una cognición individual (Collazos \& Mendoza, 2006; Dillenbourg, 1999)

\subsection{Aprendizaje colaborativo apoyado por el computador}

El Computer-Supported Collaborative Learning (CSCL) o aprendizaje colaborativo apoyado por computador es un modelo educativo que se centra en el uso de las tecnologías de información y comunicación (TIC) como herramientas para potenciar el aprendizaje colaborativo (Koschman, Kelson, Feltovich, \& Barrows, 1996). Por lo general los ambientes CSCL involucran estudiantes distribuidos en grupos que trabajan juntos sobre un problema o proyecto común (Stahl, 2004). En esta modalidad se presentan los mismos elementos del aprendizaje colaborativo, pero la dinámica varía por el uso de una herramienta que apoya el aprendizaje. Esta mediación además de conllevar cambios en la organización del aprendizaje, también reporta beneficios en el aprendizaje de los estudiantes, tales como habilidades de pensamiento crítico, autonomía en el aprendizaje y, habilidades sociales personales y de grupo (Roschelle, Pea, Hoadley, Gordin, \& Means, 2000; Tatar, Roschelle, Vahey, \& Penuel, 2003)

De acuerdo a Gros y Silva (2005), el aprendizaje colaborativo mediado por el computador conlleva dos ideas importantes. La primera es la idea de aprender en grupo, en interacción con otros. Aquí se parte de la premisa de que compartir objetivos y distribuir responsabilidades son formas deseables de aprendizaje. En segundo lugar, se enfatiza en el computador como el elemento mediador que apoya este proceso, el cual 
debe favorecer los procesos de interacción y de la solución conjunta de los problemas o tareas asignadas.

Según Liponnen (2002) la colaboración puede ser vista como una forma especial de interacción, y el CSCL analiza cómo la tecnología y la colaboración facilitan la distribución del conocimiento y el compartir experiencias entre pares y en el trabajo en grupos. Con ayuda de las TIC, los estudiantes pueden formar sus ideas, centrar su atención en la tarea y profundizar en conjunto en la solución de la misma, sin tener que estar reunidos físicamente.

El aprendizaje colaborativo apoyado en las TICs, tiene los siguientes beneficios (Calzadilla, 2002): (1) estimula la comunicación interpersonal al facilitar el intercambio de información y el diálogo y la discusión entre los estudiantes; (2) facilita el trabajo colaborativo, al permitir que los estudiantes compartan información, trabajen con documentos conjuntos y faciliten la solución de problemas y la toma de decisiones; (3) permite el seguimiento del progreso del grupo, a nivel individual y colectivo; (4) posibilita el acceso a información y contenidos de aprendizaje; y (5) facilita la gestión y administración de los estudiantes. Sin embargo, a pesar de sus potenciales beneficios, no es sencillo planificar este tipo de ambientes de aprendizaje. Como se mencionó anteriormente, no basta con poner a un grupo de estudiantes a interactuar para que se produzca aprendizaje. Se deben articular diferentes elementos para lograr los procesos conjuntos de intercambio y construcción del conocimiento, además el diseño de las actividades que promuevan este tipo de aprendizaje colaborativo deben procurar que el éxito de un individuo esté vinculado con el éxito de los demás (Collazos et al., 2007; Gros \& Silva, 2005)

\subsection{Aprendizaje ubicuo}

El aprendizaje ubicuo o u-learning es un nuevo paradigma de aprendizaje. Se dice que es una expansión de paradigmas de aprendizaje anteriores que surge a medida que se ha avanzado del aprendizaje convencional al aprendizaje electrónico (e-learning) y del e-learning al aprendizaje móvil (m-learning), y ahora al u-learning. El aprendizaje ubicuo está basado en la tecnología ubicua, cuyo rol es facilitar la construcción de un ambiente de aprendizaje que le permita a una persona aprender en cualquier lugar y en cualquier momento (Yahya, Ahmad, \& Jalil, 2010).

Yahya y otros (2010) proponen una serie de características del aprendizaje ubicuo: (1) permanencia: los usuarios nunca pierden su información a menos que decidan eliminarlos. (2) accesibilidad: la información está siempre disponible cada vez que los estudiantes necesiten utilizarla. (3) inmediatez: la información puede ser recuperada inmediatamente por los estudiantes. (4) interactividad: los estudiantes pueden interactuar con sus compañeros, profesores y expertos con eficiencia y eficacia, a través de diferentes medios de comunicación. (5) adaptabilidad: el entorno es sensitivo al contexto y se adapta a las necesidades reales de los estudiantes para proporcionarles la información adecuada. 
El u-learning no se limita a la formación recibida a través del computador o de dispositivos móviles, sino que aprovecha cualquier medio tecnológico que permita recibir información y facilite el aprendizaje. De este modo se deja de visualizar al elearning y m-learning como metodologías aisladas y conceptos separados, para pensar en un contexto u-learning, donde el aprendizaje abarca todo lo anterior y además, incluye todos los medios tecnológicos existentes que puedan ser utilizados para la enseñanza-aprendizaje (Collazos, Moreno, Yandar, Vicari, \& Coto, 2013).

Las ventajas del aprendizaje ubicuo es que permite a los estudiantes seleccionar los objetivos de aprendizaje y aplicar su estilo de aprendizaje, a la vez que les permite utilizar cualquier plataforma a su alcance y situarse en casi cualquier parte del mundo. Es decir se traslada el aprendizaje más allá del aula, hacia los ambientes de la vida diaria, apoyado en una tecnología flexible y omnipresente.

\subsection{Ingeniería de la colaboración}

Cuando las personas unen sus esfuerzos individuales para lograr un objetivo común, este esfuerzo de grupo puede resultar productivo y exitoso o improductivo y fallido, ya que el trabajo en grupo enfrenta numerosos y diversos retos (Fjermestad \& Hiltz, 2001)

Para incrementar la productividad de los equipos de trabajo, muchas veces se incorporan herramientas computacionales de apoyo, sin embargo estas tampoco garantizan el éxito del proceso, ya que se requiere conocer y ejecutar las funciones de la herramienta en el momento en que el grupo las necesite, y así poder obtener los resultados esperados (Vreede, Davison, \& Briggs, 2003).

Para lograr mayores posibilidades de éxito en los procesos colaborativos, algunos autores (Briggs, Kolfschoten, Gert-Jan, \& Douglas, 2006) han argumentado que estos deben ser explícitamente diseñados, estructurados y manejados. Este proceso de diseñar y estructurar explícitamente los procesos colaborativos se ha convertido en el eje central de una nueva área llamada Ingeniería de Colaboración (IC), en la cual "se diseñan procesos colaborativos repetitivos, los cuales se pueden transferir a grupos, usando técnicas y tecnología de colaboración (Méndez, Jiménez, Collazos, Granollers, \& Gonzalez, 2008). La Ingeniería de la Colaboración es un acercamiento al diseño de procesos colaborativos reutilizables" (Kolfschoten \& Vreede, 2007).

La Ingeniería de la Colaboración busca entonces codificar y empaquetar en unidades de conocimiento, las actividades y sus respectivas guías de ejecución, involucrando tanto los pasos a ejecutar en una herramienta computacional, como los pasos a ejecutar entre los integrantes del grupo, de forma que estas unidades, puedan ser reutilizadas fácilmente por los miembros del grupo para potenciar el rendimiento y resultados del trabajo grupal (Méndez et al., 2008; Solano \& Collazos, 2013). En la IC se destacan los patrones de colaboración y los thinkLets, que se describen a continuación. 


\subsection{Patrones de la colaboración}

La Ingeniería de la Colaboración ha identificado una serie de patrones a partir de los cuales un grupo trabaja colaborativamente hacia sus metas. Estos patrones denominados patrones de colaboración son una guía de cómo se ejecutará un proceso, y “definen la manera como los participantes de una actividad grupal van de un estado inicial a un estado final" (Kolfschoten, Briggs, \& Vreede, 2006). Cada patrón tiene sub-patrones que se pueden relacionar con las actividades, en la descripción del proceso genérico. Los patrones de colaboración son (Kolfschoten, Briggs, Appelman, \& Vreede, 2004; Méndez et al., 2008):

a) Generación: es un patrón a partir del cual el grupo crea contenido. Consiste en pasar de tener pocos a muchos conceptos que son compartidos por el grupo.

b) Reducción: el objetivo de este patrón es mantener sólo la información que cumple con un determinado criterio. Consiste en pasar de tener muchos conceptos a unos pocos que el grupo considere que requieren mayor atención.

c) Clarificación: el objetivo de este patrón es lograr el entendimiento común de conceptos manejados por el grupo. Consiste en pasar de tener un menor a un mayor conocimiento compartido de los conceptos, las palabras y frases usadas por los integrantes del grupo.

d) Organización: consiste en pasar de tener un menor a un mayor conocimiento de las relaciones entre los conceptos que el grupo esté considerando.

e) Evaluación: consiste en pasar de un menor a un mayor conocimiento del valor relativo de los conceptos bajo consideración. Este patrón tiene como efectos apoyar a la toma de decisiones y a la comunicación del grupo.

f) Construcción de Consenso: consiste en moverse de tener pocos a muchos miembros del grupo quienes estarán dispuestos a comprometerse para un objetivo.

Los patrones de colaboración son una guía de cómo se ejecutará un proceso, sin embargo, no presentan una forma detallada para guiar a un grupo de trabajo en la ejecución de un proceso, para ellos se crearon los thinkLets que son "técnicas de facilitación repetibles, transferibles y predecibles para asistir a un grupo a alcanzar el objetivo acordado" (Briggs, Vreede, \& Nunamaker, 2003).

Un proceso de colaboración consiste en una serie de actividades ejecutadas por un grupo para lograr un objetivo. Un supuesto fundamental en el diseño de procesos de colaboración, es que cada proceso consiste de una secuencia particular de thinkLets y actividades, que apoyan la creación de colaboración o patrones de colaboración entre los miembros del equipo (Acosta, 2010). Con el objetivo de ir de una fase a otra del proceso de colaboración, cada actividad puede ser apoyada por una combinación de ThinkLets.

\subsection{ThinkLets}

Un thinkLet se refiere a la unidad más pequeña de conocimiento requerido, que se necesita para ser capaces de reproducir la colaboración entre personas que trabajan hacia un objetivo común (Santanen \& Vreede, 2004). Los thinkLets pueden ser usados como unidades conceptuales de construcción en el diseño de procesos que involucran la colaboración como elemento, ya que cada thinkLet proporciona el conocimiento necesario sobre los pasos o instrucciones a seguir para implementarlos como parte del proceso grupal (Kolfschoten et al., 2006). 
Una de las grandes ventajas de los thinkLets es que al diseñar procesos colaborativos se pueden emplear soluciones conocidas y no invertir esfuerzos en inventar y probar nuevas (Solano \& Collazos, 2013), por lo tanto, el enfoque de trabajo cambia de inventar y probar soluciones, a escoger soluciones conocidas y probadas, lo que puede reducir tanto el esfuerzo como el riesgo de desarrollar e implementar procesos grupales (Kolfschoten et al., 2004). Por ejemplo, un thinkLet en el que un grupo vota sobre un conjunto de ideas puede crear un modelo de evaluación y dará lugar a una clasificación del conjunto de ideas basadas en el criterio de votación. Un thinkLet en el que el grupo selecciona una serie de ideas que debe abordarse en una discusión de grupo posterior, crea un patrón de evaluación, así como un patrón de reducción (Kolfschoten, Vreede, \& Briggs, 2010).

Los thinkLets se detallan completamente y son modificables. Pueden usarse para construir nuevos grupos de procesos. Son recetas que pueden ser utilizadas por usuarios sin mucha experiencia, dado que pueden ser fácilmente aprendidos, recordados y se pueden adaptar fácilmente a un diseño de proceso (Briggs et al., 2003). En la Tabla 1 se presenta una descripción general de algunos thinklets (Méndez et al., 2008):

Tabla 1. ThinkLets

\begin{tabular}{|l|l|l|}
\hline $\begin{array}{c}\text { Nombre del } \\
\text { thinkLet }\end{array}$ & $\begin{array}{c}\text { Patrón de } \\
\text { colaboració } \\
\text { n }\end{array}$ & \multicolumn{1}{|c|}{ Información general } \\
\hline LeafHopper & Generación & $\begin{array}{l}\text { Los participantes empiezan con una lista de varios } \\
\text { tópicos de discusión. Cada ítem de la lista tiene } \\
\text { asociado una ventana de comentarios. Cada } \\
\text { participante hace su aporte dependiendo del que más le } \\
\text { interese y en el cual tenga mayor experiencia }\end{array}$ \\
\hline $\begin{array}{l}\text { FreeBrainsto } \\
\text { rm }\end{array}$ & Generación & $\begin{array}{l}\text { Los integrantes del equipo generan ideas en respuesta a } \\
\text { una pregunta o sugerencia. Cada participante empieza } \\
\text { sobre una página diferente. Una vez el participante } \\
\text { termina de realizar sus comentarios, debe enviar esta } \\
\text { página a los demás miembros del grupo de trabajo. El } \\
\text { sistema recupera otra página al azar. Los participantes } \\
\text { construyen en esta nueva página los comentarios } \\
\text { respectivos. }\end{array}$ \\
\hline $\begin{array}{l}\text { BroomWago } \\
\mathrm{n}\end{array}$ & Reducción & $\begin{array}{l}\text { Los integrantes del grupo escogen algunos ítems de un } \\
\text { listado general, dependiendo del número que se le } \\
\text { solicite }\end{array}$ \\
\hline $\begin{array}{l}\text { Concentratio } \\
\mathrm{n}\end{array}$ & Organización & $\begin{array}{l}\text { Se guía al grupo para eliminar duplicados, combinar } \\
\text { ideas y reescribir ideas no claras. }\end{array}$ \\
\hline
\end{tabular}

Fuente: (Méndez et al., 2008)

Las investigaciones y experiencias de campo sugieren que dado un proceso de colaboración, los thinklets forman el $80 \%$ del diseño de las acciones que un grupo debe ejecutar y que el otro 20\% necesitan ser personalizadas (Acosta, 2010). Desde este punto de vista estos han llegado a ser un lenguaje de patrones poderoso, y los ingenieros usan los nombres de los thinkLets para describir y comunicar procesos complejos y sofisticados en forma más compacta (Briggs et al., 2003). 
Cada thinklet, cuenta con la siguiente información (Briggs et al., 2003): un nombre representativo relacionado con los patrones que crea, criterios para decidir cuándo escogerlo o no, entradas y salidas, pasos que lo conforman, una historia exitosa que ayuda a clarificar las circunstancias bajo las cuales el thinklet es útil y una explicación del nombre. A manera de ejemplo, la Tabla 2 muestra la descripción para el thinkLet LeaftHopper (Méndez et al., 2008):

\section{Tabla 2. Descripción del Thinklet LeafHopper}

\begin{tabular}{|l|l|}
\hline \multicolumn{2}{|c|}{ Thinklet LeafHopper } \\
\hline $\begin{array}{l}\text { Escoger este } \\
\text { thinklet }\end{array}$ & $\begin{array}{l}\text { Cuando sepa por adelantado que el equipo va a dar ideas sobre varios } \\
\text { tópicos a la vez } \\
\text { Cuando los participantes tengan diferentes niveles de interés o experticia en } \\
\text { los distintos tópicos } \\
\text { Cuando no sea importante asegurar que todos los participantes contribuyan a } \\
\text { todos los tópicos }\end{array}$ \\
\hline $\begin{array}{l}\text { No escoger } \\
\text { este ThinkLet }\end{array}$ & $\begin{array}{l}\text { Cuando es importante asegurar que cada persona aporte sobre todos los } \\
\text { tópicos }\end{array}$ \\
\hline $\begin{array}{l}\text { Información } \\
\text { general }\end{array}$ & $\begin{array}{l}\text { Los participantes empiezan con una lista de varios tópicos de discusión. } \\
\text { Cada ítem de la lista tiene asociado una ventana de comentarios. Cada } \\
\text { participante hace su aporte dependiendo del que más le interese y en el cual } \\
\text { tenga mayor experiencia. } \\
\text { Entradas: Una lista de tópicos que serán dirigidos por el equipo } \\
\text { Salidas: Un conjunto de comentarios organizados por tópicos de discusión }\end{array}$ \\
\hline Configuración & $\begin{array}{l}\text { Crear una lista con los tópicos que serán discutidos } \\
\text { Los participantes pueden contribuir con comentarios del tópico } \\
\text { Los participantes no pueden contribuir con nuevos tópicos } \\
\text { Las contribuciones podrían ser anónimas }\end{array}$ \\
\hline Pasos & $\begin{array}{l}\text { Presentar la información sobre los diferentes tópicos para que el grupo dé } \\
\text { sus contribuciones } \\
\text { Informar a los participantes sobre el tipo de ideas con las que puede } \\
\text { contribuir } \\
\text { Sugerir a los participantes que empiecen trabajando en algunos de los } \\
\text { tópicos en los cuales tenga más interés y/o experiencia } \\
\text { Solicitar que una vez hayan realizado alguna contribución debe enviarla a } \\
\text { los demás integrantes del grupo } \\
\text { Pedir que se recorran cada uno de los otros tópicos para leer y hacer } \\
\text { comentarios sobre las contribuciones de los demás } \\
\text { Continuar moderando la actividad hasta que se llegue a un límite de tiempo } \\
\text { previamente determinado o hasta que ya no se tengan más comentarios }\end{array}$ \\
\hline $\begin{array}{l}\text { Algunas veces el equipo debe discutir varios tópicos más o menos } \\
\text { simultáneamente. Con LeafHopper no necesariamente todos los } \\
\text { participantes verán todos los tópicos. }\end{array}$ \\
\hline Observaciones
\end{tabular}
Fuente: (Méndez et al., 2008)

\section{El modelo propuesto: U-CSCL}

Es bien sabido que la incorporación de las tecnologías de la información y de las comunicaciones (TIC) a los entornos educativos ha cambiado dichos entornos de múltiples maneras, por ejemplo, la forma en que se comunican los docentes y los estudiantes, y las maneras de interactuar con la información y los contenidos. Se han creado entornos más flexibles para el aprendizaje, eliminando las barreras espacio-

Modelo Colaborativo y Ubicuo para apoyar los procesos de enseñanza-aprendizaje a nivel Iberoamericano. Mayela Coto, César Collazos y Sonia Mora. 
temporales para la interacción entre los diversos actores, e incrementando las modalidades de comunicación, y de entornos interactivos, que favorecen tanto el aprendizaje independiente como el aprendizaje colaborativo, y ofrecen nuevas posibilidades para organizar la actividad docente (Cabero \& Llorente, 2008).

El modelo u-CSCL (Figura 1) es un modelo de aprendizaje, desarrollado a nivel Iberoamericano, que está constituido primordialmente por las mejores prácticas de dos formas de aprendizaje: (1) el aprendizaje ubicuo (u-learning) que utiliza actividades formativas apoyadas en la tecnología, accesibles en cualquier lugar y disponible en distintos canales al mismo tiempo; y (2) CSCL que es el aprendizaje colaborativo apoyado por herramientas computacionales, enfocado en ambientes educativos (Collazos et al., 2013).

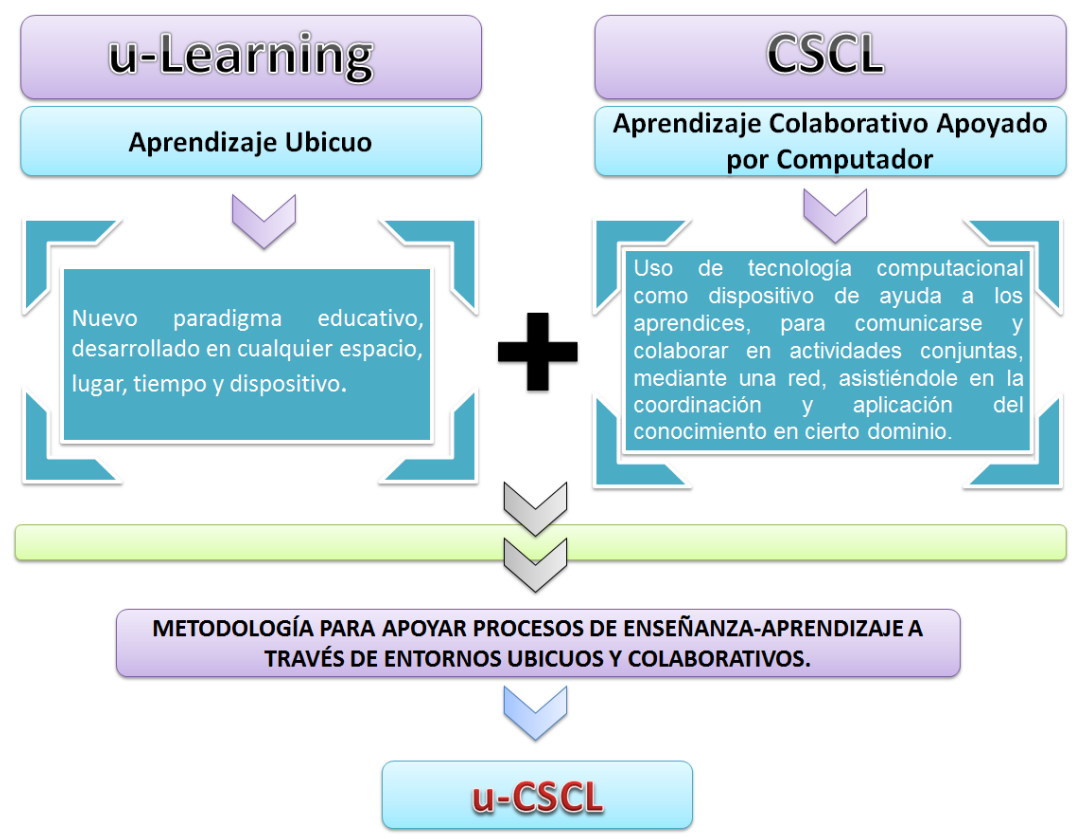

Figura 1: Conformación del enfoque u-CSCL.

Fuente: (Collazos et al., 2013)

Al igual que otros modelos educativos, el modelo u-CSCL, incluye cinco componentes principales: 1. Docentes, 2. Estudiantes, 3. Materiales de estudios (contenidos, actividades colaborativas, contenedor de objetos de aprendizaje), 4. Plataforma Tecnológica (sistemas de gestión de aprendizaje, ambientes virtuales de aprendizaje), 5. Servicios de acceso (Collazos et al., 2013).

En la Figura 2 se presenta una propuesta inicial del modelo u-CSCL, donde los profesores (I) tienen a disposición un entorno colaborativo de apoyo a la generación de contenidos (II) y actividades (III). Esto implica que un conjunto de profesores geográficamente dispersos pueden diseñar sus cursos de manera colaborativa. La parte concerniente a las Actividades (III), involucra un esquema que incluya técnicas de aprendizaje colaborativo, de tal forma que los estudiantes (VII) trabajen 
colaborativamente para resolver las tareas asignadas. La etapa de evaluación y seguimiento consecuentemente se puede realizar de forma colaborativa.

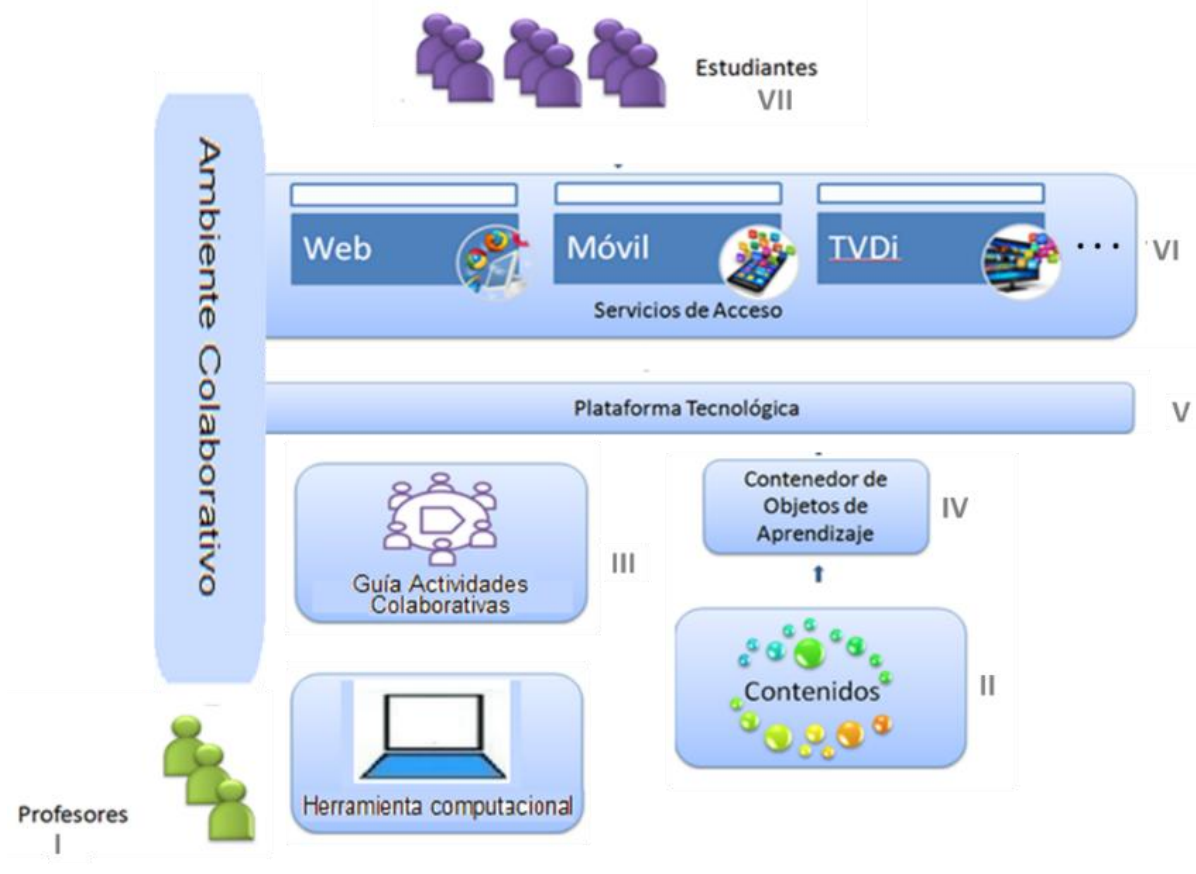

Figura 2: Esquema inicial del modelo u-CSCL.

Fuente: (Collazos et al., 2013).

Para la incorporación de los contenidos (II) y las actividades (III) se definen estándares de representación de contenido educativo y procesos de aprendizaje en el modelo, estableciendo un contenedor de Objetos de Aprendizaje (IV). Para la creación de los contenidos por parte de los docentes, el modelo contempla el desarrollo de una plataforma tecnológica que permita la elaboración de los cursos de manera colaborativa, así como una guía que oriente el proceso. Este proceso de diseño colaborativo de contenidos se basa en los conceptos de la ingeniería de la colaboración. En la parte de la plataforma tecnológica (V) el modelo está basado en el diseño de una multiplataforma de gestión de conocimiento que integre componentes de m-learning, y e-learning para prestar servicios a través de un modelo de aprendizaje colaborativo (VI). Finalmente, los estudiantes (VII) podrán acceder fácilmente a la información (contenidos y actividades) desde cualquier parte y dispositivo (Collazos et al., 2013).

\subsection{Metodología para el desarrollo de procesos colaborativos (MDPC)}

La metodología utilizada para el desarrollo de las actividades colaborativas es la MDPC (Kolfschoten \& Vreede, 2007; Méndez et al., 2008; Solano \& Collazos, 2013) la cual permite obtener la especificación colaborativa de un proceso a partir de la identificación de las actividades recurrentes o destacadas. De esta manera, las actividades especificadas de forma colaborativa promueven la comunicación, la coordinación y la negociación, con el fin de aumentar la productividad en la realización de dichas actividades. 
En forma paralela a esta metodología se utilizó también la Guía CSCoLAD (Ramírez \& Bolaños, 2013) la cual proporciona una alternativa genérica para dar solución al diseño de actividades de aprendizaje colaborativo de cualquier índole y nivel educativo. Para cada actividad se detalla el responsable y las entradas y salidas. Como entradas se identifican las variables que son requisitos para realizar la actividad y como salidas se obtiene el resultado de la actividad que pueden ser, a su vez, insumos para actividades posteriores. La guía incluye una serie de recomendaciones dirigidas a los docentes, que involucra las fases de diseño, ejecución y prueba de la actividad.

En la Figura 3 se muestran las distintas fases de la metodología, las cajas en gris de la parte superior son las entradas que se requieren en cada una de las fases y las cajas en celeste de la parte inferior, describen la salida de cada fase. El procedimiento a seguir en cada fase es el siguiente (Kolfschoten et al., 2010; Solano \& Collazos, 2013):

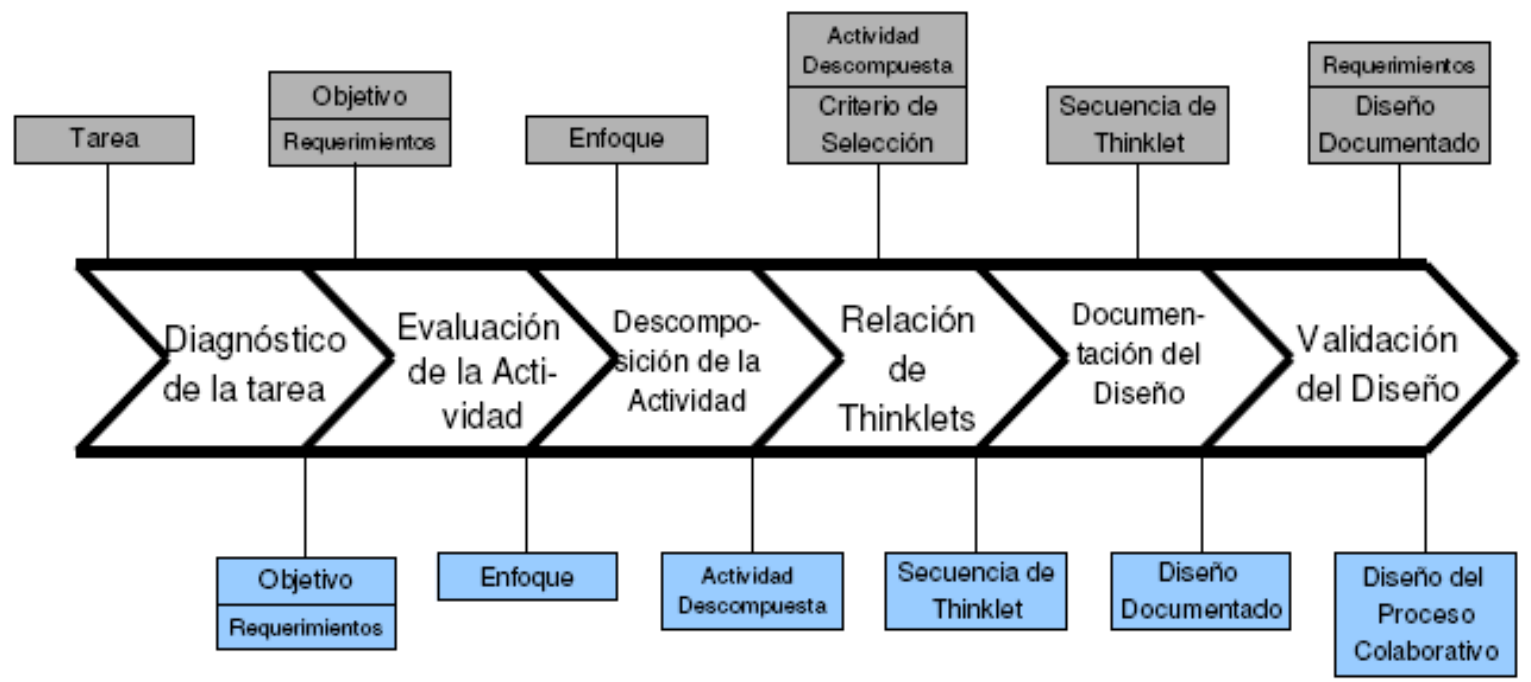

Figura 3: Metodología para el desarrollo de procesos colaborativos.

Fuente:(Kolfschoten \& Vreede, 2007)

- Fase 1 - Diagnóstico de la tarea: se realiza una descripción detallada del proceso o tarea objeto de estudio. Dicha descripción debe incluir información como los entregables, requisitos, participantes y demás características relevantes del proceso.

- Fase 2 - Evaluación de la actividad: para el proceso objeto de estudio, deben identificarse las actividades generales que lo componen y determinar la secuencia entre ellas.

- Fase 3 - Descomposición de la actividad: descripción de las sub-actividades que componen cada una de las actividades generales identificadas en la Fase 2. La división de las sub-actividades permite identificar cuáles se realizarían de forma colaborativa para asociarles uno o más patrones de colaboración. 
- Fase 4 - Relación de thinkLets: en esta fase se relacionan los thinkLets a las subactividades definidas como colaborativas. Para seleccionar los thinkLets deben considerarse los siguientes aspectos (Collazos \& Solano, 2014):

- Criterios para decidir cuándo usar o no el thinkLet

- Pasos que conforman el thinkLet

- Mapa de selección: indica el tipo de relación entre los thinkLets

- Fase 5 - Documentación del diseño: a partir de la información obtenida en las fases anteriores, en esta fase se generan los siguientes elementos definidos en la IC:

- Agenda detallada: documento que presenta de manera clara y detallada la información de las actividades que forman parte del proceso diseñado. La agenda detallada consta de la siguiente información: número de secuencia de la actividad, descripción de la actividad, entregables, thinklet y patrón de colaboración que está siendo utilizado, descripción detallada de los pasos requeridos para ejecutar la actividad (proceso colaborativo) y personas que participan en la realización de la actividad.

- Modelo de facilitación del proceso (MFP): es utilizado para mostrar el flujo del proceso y los elementos tales como thinkLet, número de secuencia, patrón de colaboración y nombre, relacionados con cada una de las actividades que conforman el proceso. En el MFP se representa cada actividad como un rectángulo que se divide en cuatro campos (Figura 4). En la parte superior izquierda se indica el número de secuencia, correspondiente con la agenda detallada. El campo más grande contiene un nombre de la actividad. El campo de la izquierda tiene el nombre del patrón de colaboración asociado a la actividad y el nombre del thinklet se ubica en el campo superior (Kolfschoten \& Vreede, 2009). En un MFP que consta de varias actividades, las flechas indican la dirección del flujo del proceso.

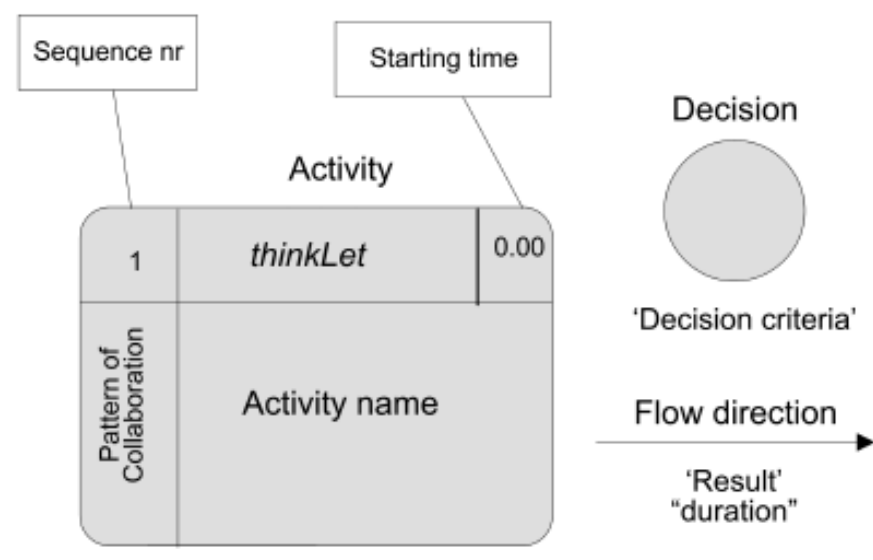

Figura 4: Estructura del Modelo de Facilitación del

Proceso.Fuente:(Kolfschoten \& Vreede. 2009)

- Fase 6 - Validación del diseño: en esta fase se valida la especificación del proceso colaborativo. La metodología ofrece las siguientes formas de validación (Collazos \& Solano, 2014):

- Prueba piloto: Se ejecuta el método de evaluación colaborativo diseñado para evaluar la efectividad del proceso. El objetivo es verificar si la 
ejecución del método se puede llevar a cabo en el tiempo estimado y con los recursos definidos.

- Recorrido: Con algunos de los posibles participantes, se realiza la evaluación del mismo para identificar falencias y dificultades durante la ejecución.

- Simulación: El equipo de trabajo que diseñó el método de evaluación colaborativo da respuesta a una serie de preguntas, como por ejemplo: ¿Estos pasos son suficientes?, ¿Está toda la información disponible?, entre otras. Esta validación prueba la lógica del diseño y las respuestas generadas servirán de referente para realizar las mejoras respectivas.

- Revisión: Se genera discusión sobre el diseño realizado, entre el equipo de trabajo y los potenciales usuarios que ejecutarán el método de evaluación colaborativo. Discutir el diseño revelará diferentes perspectivas, de esta forma se identifican partes del diseño ineficientes.

\subsection{Implementación de la metodología (MDCP)}

En la Figura 5 se esquematiza el procedimiento que se siguió para el diseño de las actividades colaborativas para los docentes, basados en la metodología anteriormente descrita (MDPC).

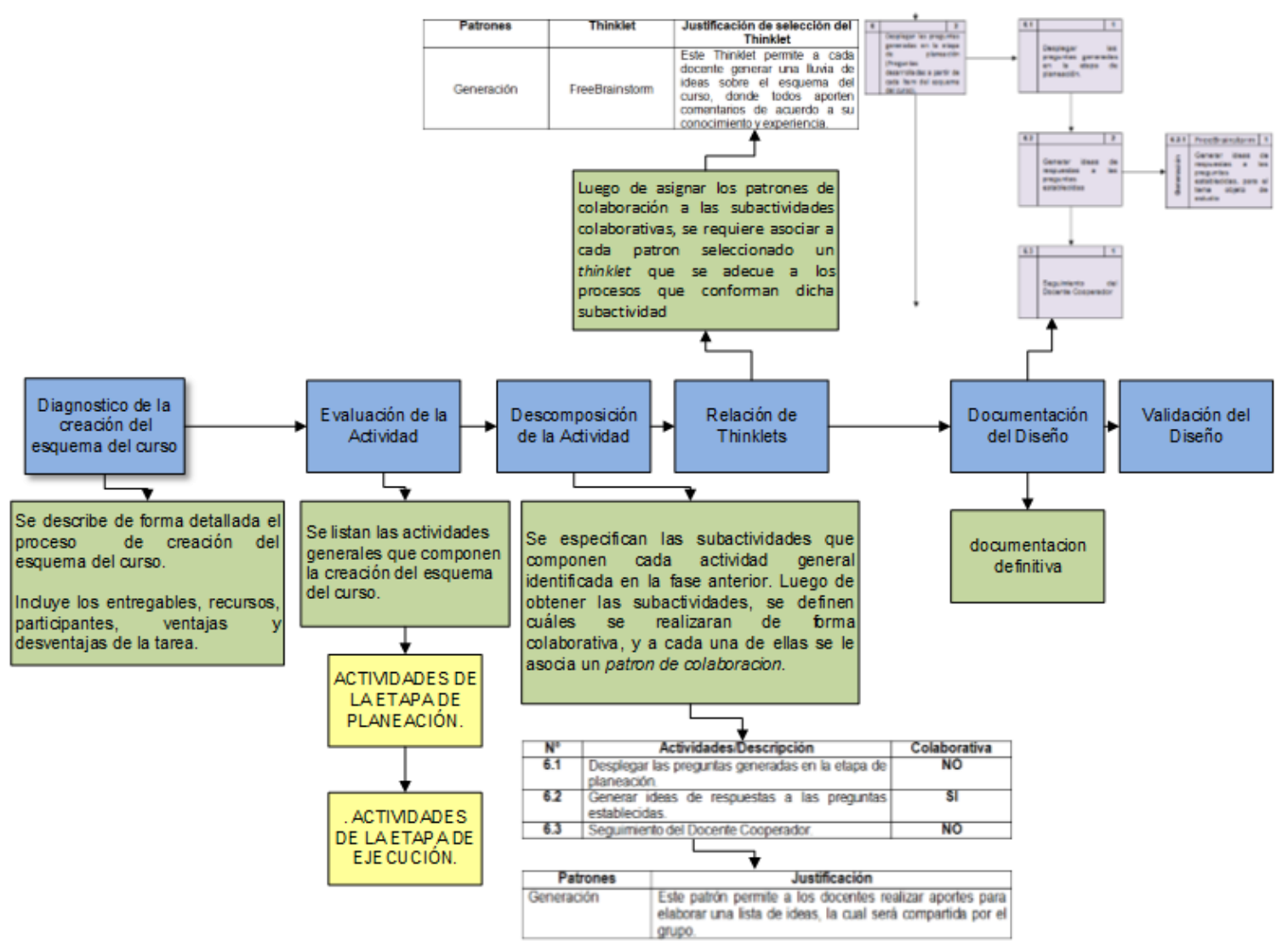

Figura 5: Esquema MDPC

Fuente: (Yandar \& Moreno, 2015)

Como se mencionó anteriormente, esta metodología describe de forma detallada el proceso objeto de estudio y ayuda a determinar qué actividades implementar colaborativamente, a la vez que guía su desarrollo. Incluye entregables, recursos, 
participantes, ventajas y desventajas de cada actividad. Cada una de estas se divide en sub-actividades, se realizan asociaciones de patrones a las sub-actividades y relaciones de thinkLets a cada sub-actividad (Yandar \& Moreno, 2015).

A manera de ejemplo, la Tabla 3, muestra un segmento de la actividad colaborativa que realizan los docentes para identificar los aspectos más importantes de un curso:

Tabla 3. Actividad colaborativa entre docentes

\begin{tabular}{|c|c|c|}
\hline \multicolumn{3}{|c|}{$\begin{array}{l}\text { Nombre actividad: Determinar por medio de una herramienta colaborativa los } \\
\text { aspectos más importantes del esquema del curso. }\end{array}$} \\
\hline $\mathrm{N}^{\mathrm{o}}$ & Actividades/Descripción & Colaborativa \\
\hline 7.1 & $\begin{array}{l}\text { Plantear los aspectos más importantes de cada uno de los } \\
\text { ítems del esquema del curso }\end{array}$ & SI \\
\hline 7.2 & Seguimiento del docente líder. & NO \\
\hline \multicolumn{3}{|c|}{$\begin{array}{l}\text { Participantes: Docente, docente cooperador. } \\
\text { Tiempo de duración estimado: } 1 \text { Día. }\end{array}$} \\
\hline \multicolumn{3}{|c|}{ Sub-actividad: Generar ideas de respuestas a las preguntas establecidas } \\
\hline \multicolumn{3}{|c|}{$\begin{array}{l}\text { Actividades relacionadas: } 2 \text { (Elaborar las preguntas correspondientes a los ítems } \\
\text { definidos, estas irán planteadas en el chat). } \\
\text { Descripción: Los docentes asignados definen una serie de ideas generales de respuestas } \\
\text { correspondiente al tema objeto de estudio. } \\
\text { Entradas: Pregunta sobre la cual se desea obtener información. } \\
\text { Resultados esperados: lista de ideas generales para elaborar el esquema correspondiente } \\
\text { al tema objeto de estudio. } \\
\text { Participantes: Docente, docente cooperador. }\end{array}$} \\
\hline Patrones & \multicolumn{2}{|l|}{ Justificación } \\
\hline Generación & \multicolumn{2}{|c|}{$\begin{array}{l}\text { Este patrón permite a los docentes realizar aportes para } \\
\text { elaborar una lista de ideas, la cual será compartida por el } \\
\text { grupo. }\end{array}$} \\
\hline
\end{tabular}

Fuente: (Yandar \& Moreno, 2015)

La Figura 6 presenta de forma gráfica el procedimiento que se siguió para el diseño de las actividades colaborativas para los docentes utilizando la guía CSCoLAD (Ramírez \& Bolaños, 2013). El ejemplo ilustra la generación del esquema del curso Liderazgo Participativo. 


\section{Actividad Colaborativa usando la guía CSCoLAD}

Actividad Modelada: Generar Esquema Del Curso

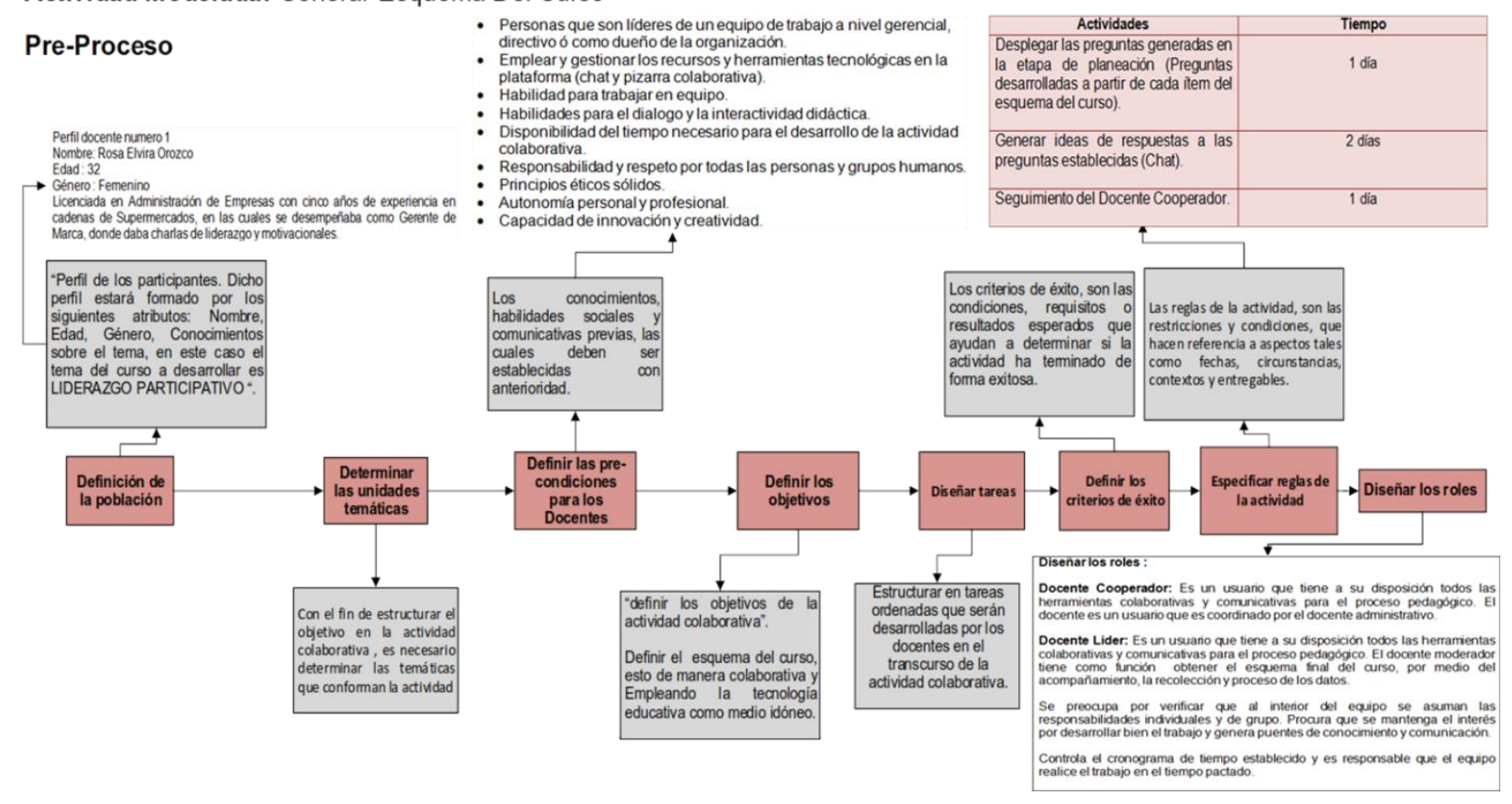

Figura 6: Generación esquema del curso "Liderazgo Participativo". Fuente: (Ramírez \& Bolaños, 2013)

\subsection{Diseño de la plataforma tecnológica}

Un entorno de aprendizaje apoyado por tecnología, como el u-CSCL, requiere de una dimensión tecnológica y una dimensión pedagógica que se interrelacionen y potencien entre sí. La dimensión tecnológica está representada por las herramientas o aplicaciones informáticas con las que está construido el entorno de aprendizaje, y la dimensión pedagógica está representada por el proceso de enseñanza-aprendizaje que se desarrolla en su interior. Esta dimensión facilita la interacción que se genera entre el docente y los estudiantes a partir de las actividades de aprendizaje. Ambas dimensiones proponen un ambiente de trabajo compartido para la construcción del conocimiento con base en la participación activa y la cooperación de todos los actores del proceso (Salinas, 2011).

\subsubsection{Dimensión pedagógica}

La dimensión pedagógica del modelo consta de dos etapas importantes. La primera es la etapa de Definición y Diseño (Figuras7a, 7b), donde los docentes de manera conjunta y colaborativamente crean "El esquema del curso" y el "Esquema teórico": enfocado a lo que se debe enseñar y qué debe aprender el alumno; la concepción del desarrollo: cómo aprende el alumno; la metodología que se utiliza: cómo enseñar y cómo lograr que el alumno aprenda; la evaluación de los conocimientos: cómo realimentar los procesos de enseñanza aprendizaje; y por último la relación docenteestudiante: cuál es el rol de cada uno y cómo debe ser su interacción. 


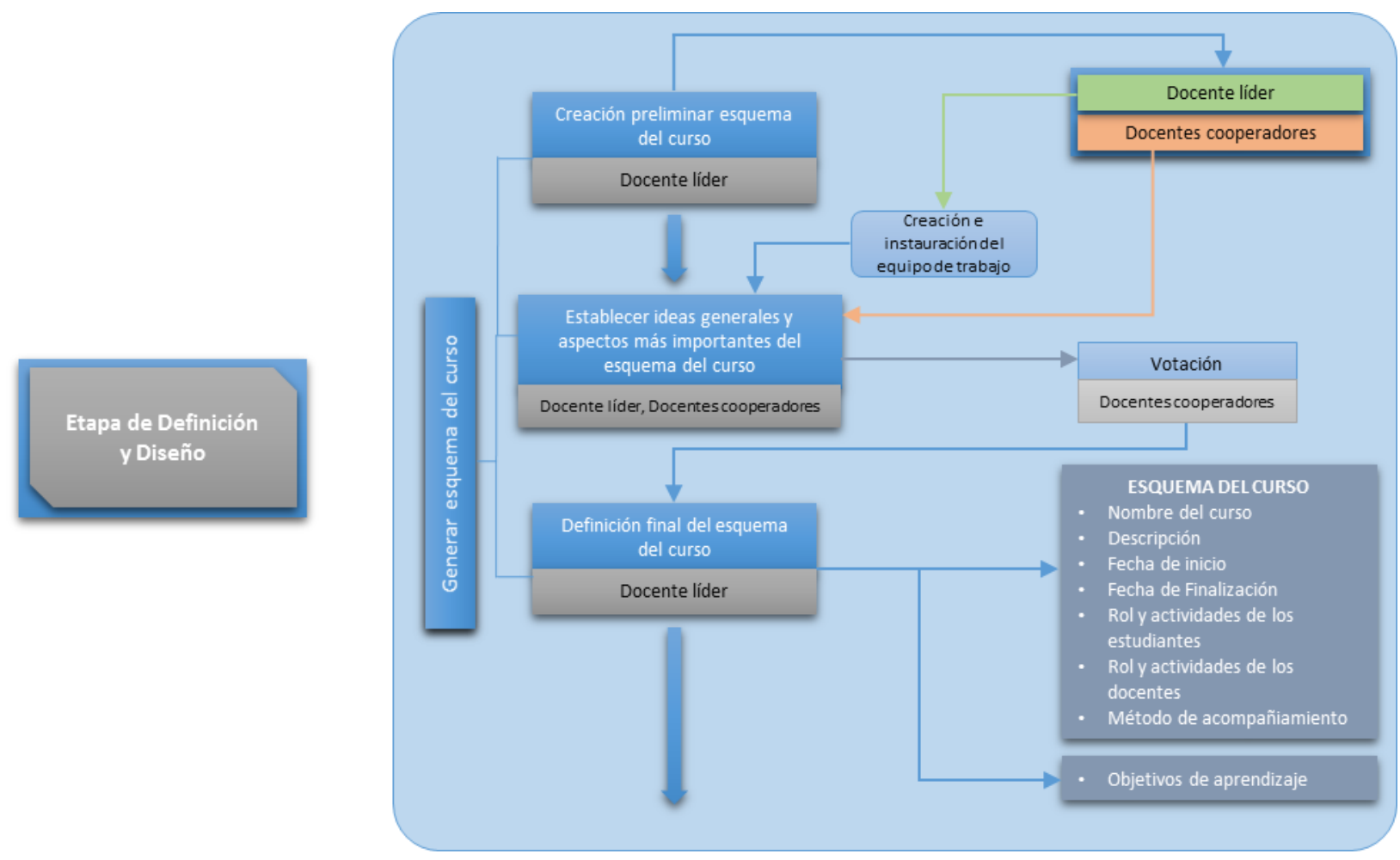

Figura 7a: Elementos pedagógicos Plataforma u-CSCL. Fase Definición y Diseño Fuente: (Yandar \& Moreno, 2015)

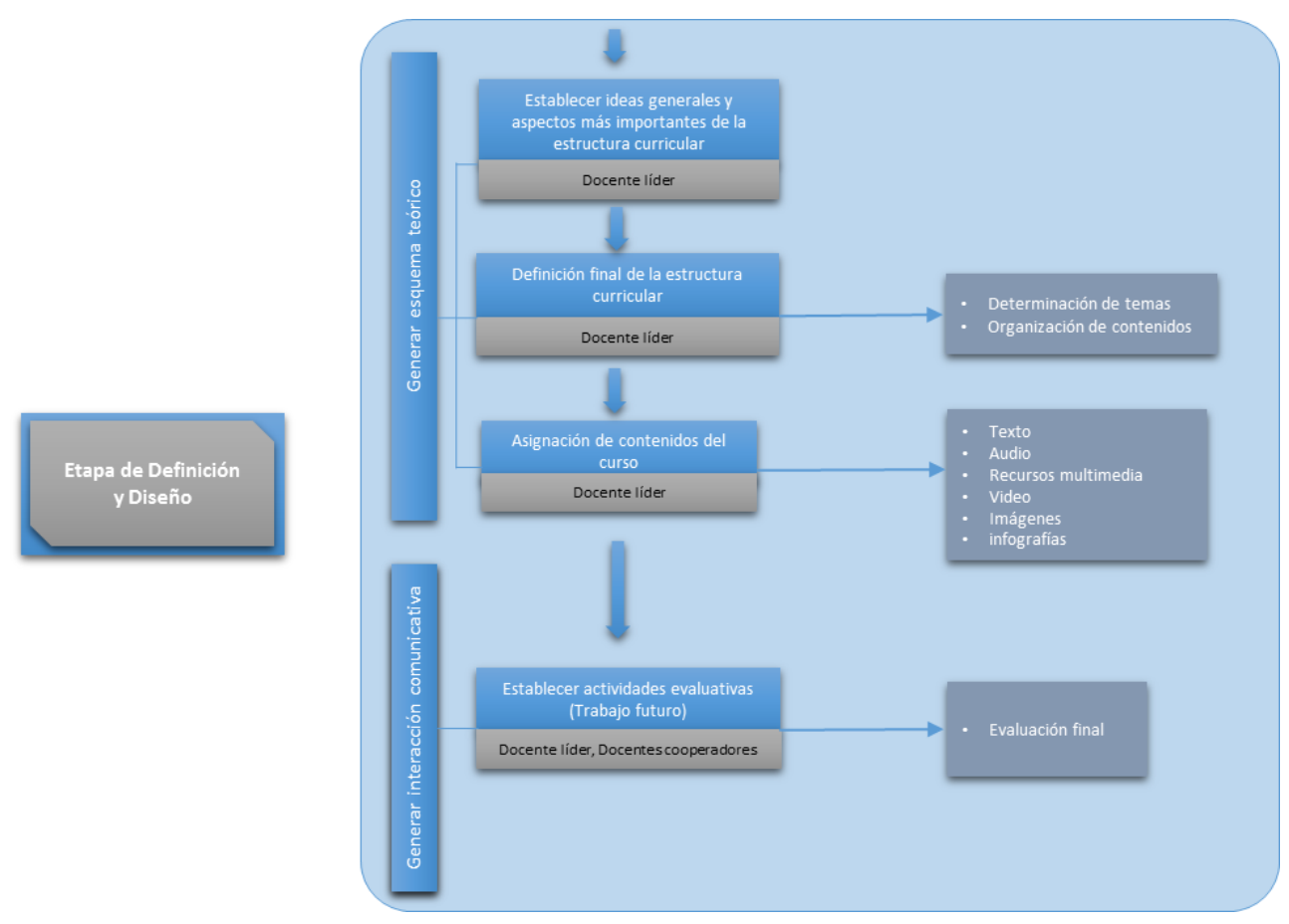

Figura 7b: Elementos pedagógicos Plataforma u-CSCL. Fase Definición y Diseño Fuente: (Yandar \& Moreno, 2015)

Modelo Colaborativo y Ubicuo para apoyar los procesos de enseñanza-aprendizaje a nivel Iberoamericano. Mayela Coto, César Collazos y Sonia Mora. 
La segunda etapa es la de "Desarrollo del curso" (Figura 8), en este los principales protagonistas son los estudiantes, los cuales desarrollarán las actividades colaborativas e individuales propuestas en el curso, es aquí donde el modelo pedagógico busca que los participantes se involucren, realicen aportes y actúen, propiciando así el desarrollo del pensamiento y la construcción del conocimiento, de tal manera que los medios tecnológicos, logren la mediación necesaria para que pueda efectuarse una actividad productiva que permita lograr una experiencia de aprendizaje completa.
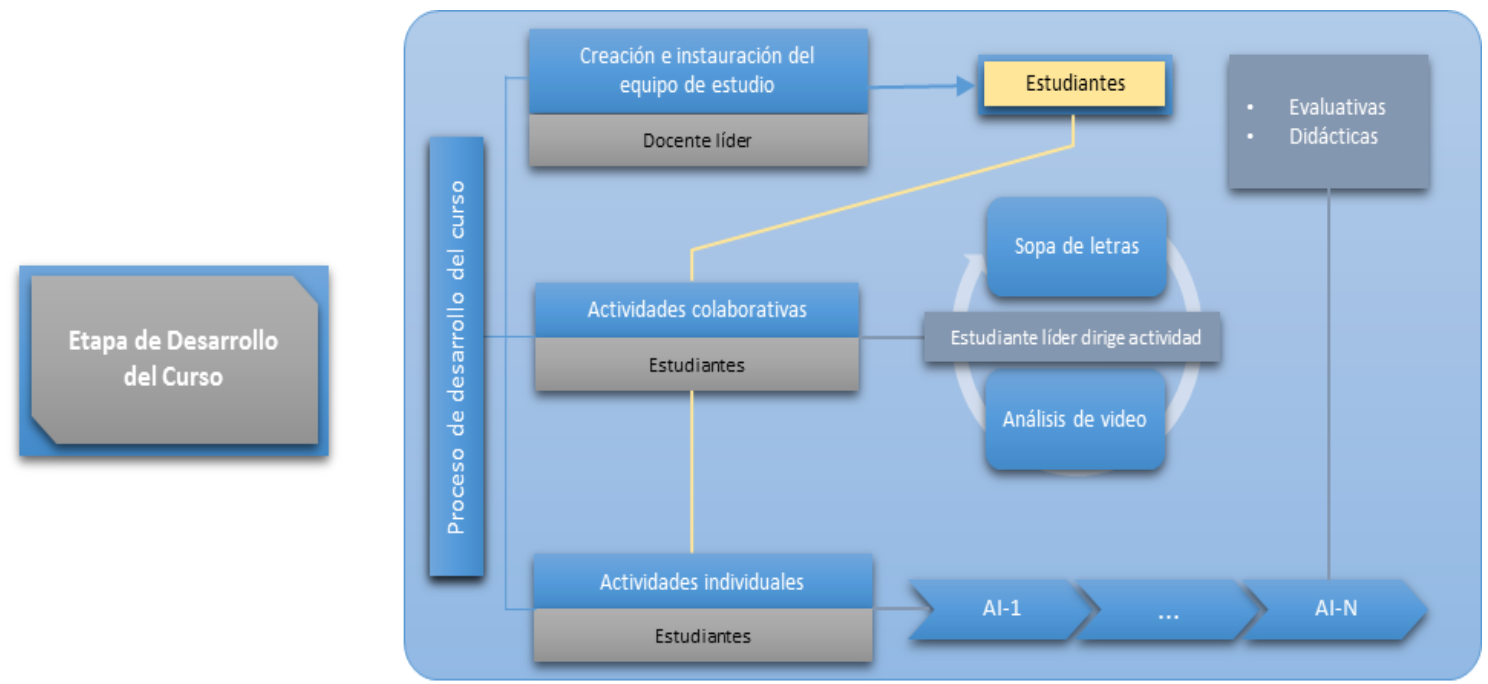

Figura 8: Elementos pedagógicos Plataforma u-CSCL. Fase Desarrollo Fuente: (Yandar \& Moreno, 2015)

\subsubsection{Dimensión tecnológica}

Para la dimensión tecnológica se hizo una revisión de diferentes plataformas tecnológicas que soportan la creación de cursos masivos, similares a los MOOCs como Coursera, MiriadaX, Udacity, entre otros. Se tomaron aspectos de dichas herramientas para diseñar una plataforma propia, en la cual se integran los aspectos colaborativos, que tanto a nivel docente como a nivel estudiantil, es el elemento diferenciador con respecto a las plataformas existentes estudiadas, de ahí la necesidad de desarrollar algo que realmente se ajuste al nuevo modelo. La plataforma permite que un conjunto de profesores geográficamente dispersos pueden diseñar un curso de manera colaborativa, utilizando medios como una pizarra colaborativa y el chat, y en cuanto a los estudiantes, estos podrán desarrollar el curso de manera conjunta y colaborativa por medio de actividades diseñadas para este fin.

El desarrollo de la plataforma se hizo mediante la metodología de desarrollo ágil $\mathrm{XP}$, por cuanto esta es una metodología para equipos de trabajo pequeño o mediano, desarrollada por medio de iteraciones, facilitando que se actualicen los requerimientos y necesidades de forma rápida, constante y permitiendo que el software sea evaluado en un ambiente real. La plataforma colaborativa se está implementando mediante el framework de desarrollo web Django, basado en el lenguaje Python, aprovechando de esta forma las ventajas de un ambiente de aprendizaje colaborativo con los beneficios de la computación ubicua y los nuevos instrumentos y recursos tecnológicos, permitiendo 
romper las barreras de espacio y tiempo que existen en las aulas de clase tradicionales (Yandar \& Moreno, 2015).

Las Figuras 9, 10, 11y 12 presentan algunas interfaces de la versión preliminar de la herramienta diseñada. En la actividad colaborativa docente (Figura 9) se despliega un formulario preliminar del esquema de un curso desarrollado por el líder de la actividad. El docente líder tiene la opción de invitar a participar a los docentes cooperadores (ingresando el correo electrónico) para realizar el desarrollo del curso como una actividad colaborativa entre docentes. Al correo de cada docente cooperador invitado, llega la siguiente información (Figura 10), que incluye el nombre del curso, descripción del curso, objetivos y tema del curso, esto para que los docentes cooperadores cuenten con información previa antes de realizar la actividad colaborativa. En la invitación existe una opción para aceptar ser un docente cooperador, la cual dirigirá a registrar al docente a la plataforma.

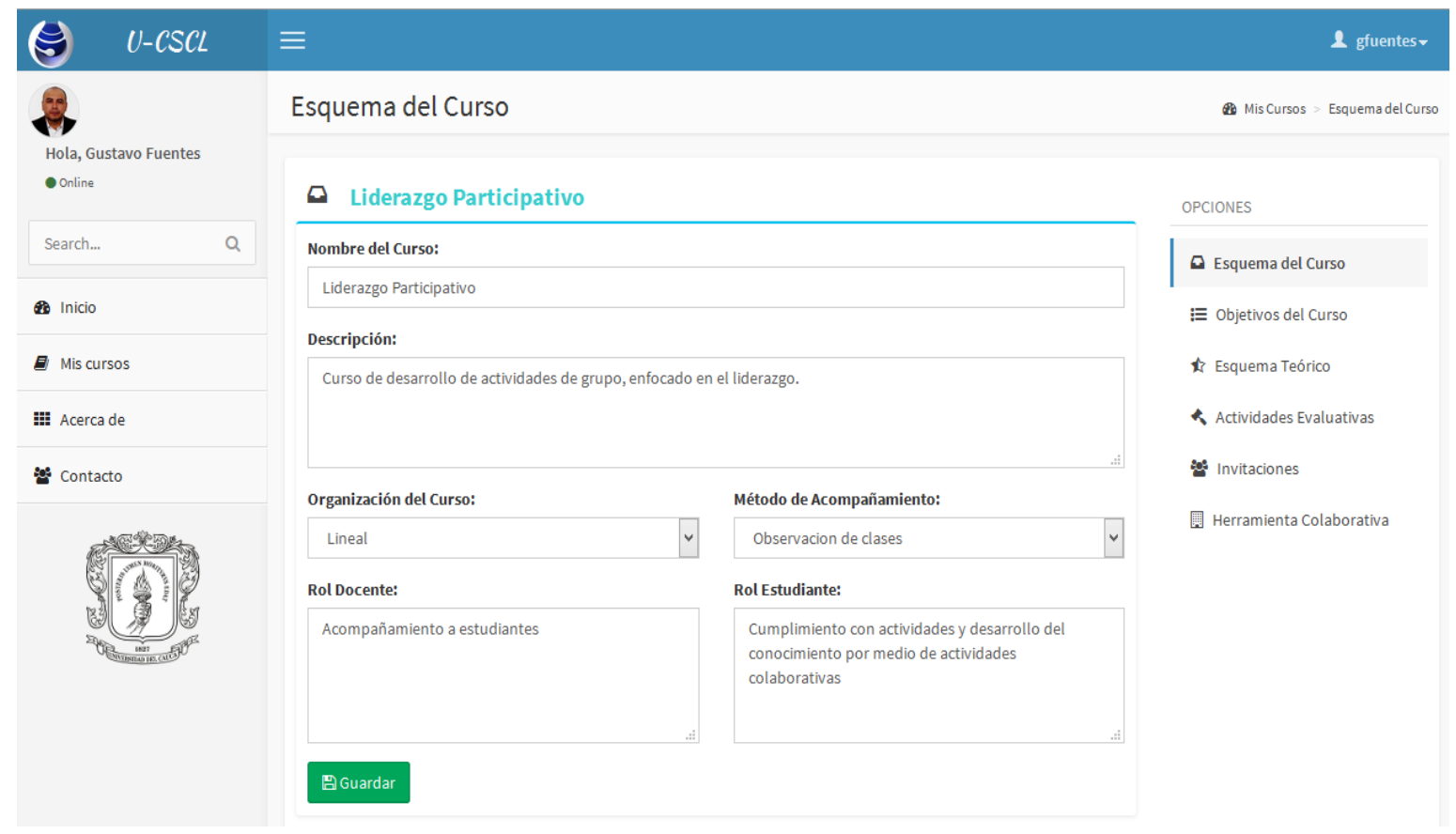

Figura 9: Formulario para crear cursos Fuente:(Yandar \& Moreno, 2015) 


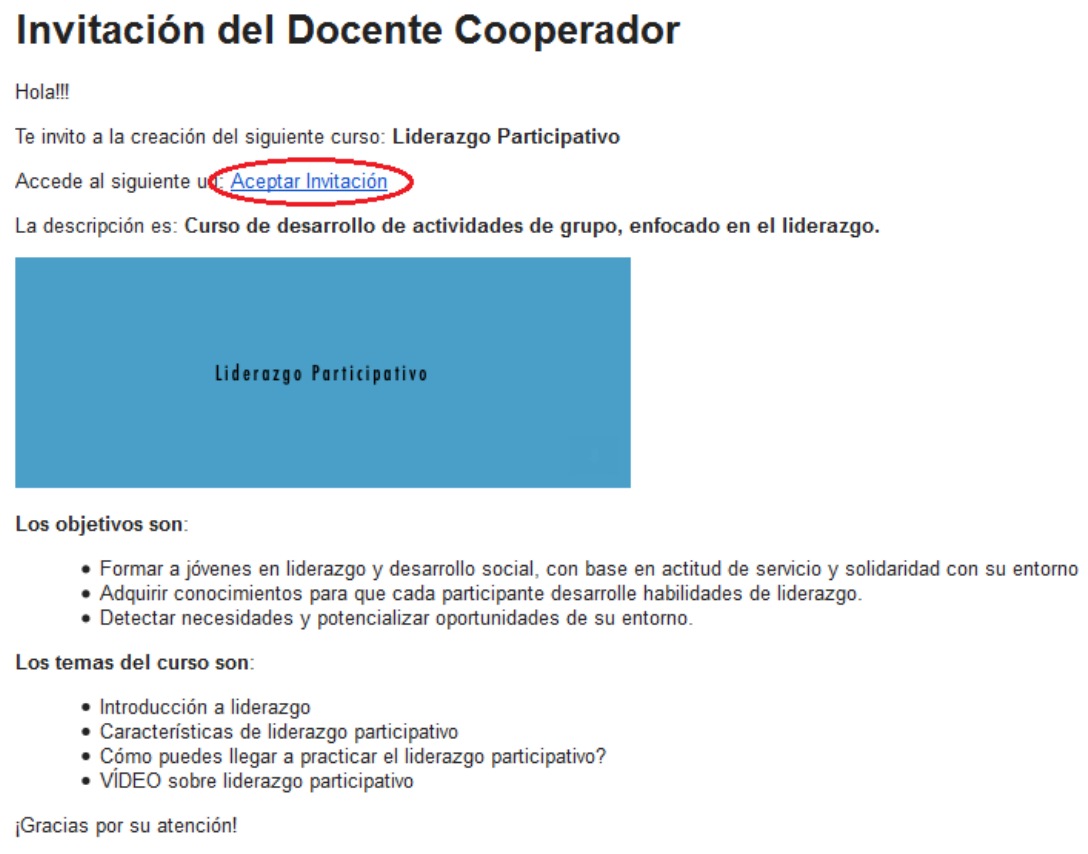

Figura 10: Invitación a los docentes cooperadores Fuente:(Yandar \& Moreno, 2015)

Posteriormente, los docentes pueden desarrollar el curso de manera tal, que cada uno de ellos pueda definir una serie de ideas generales correspondientes al esquema a trabajar, esto mediante una pizarra colaborativa; y el consenso de las ideas principales se realiza por medio de una votación (ver Figura 11).

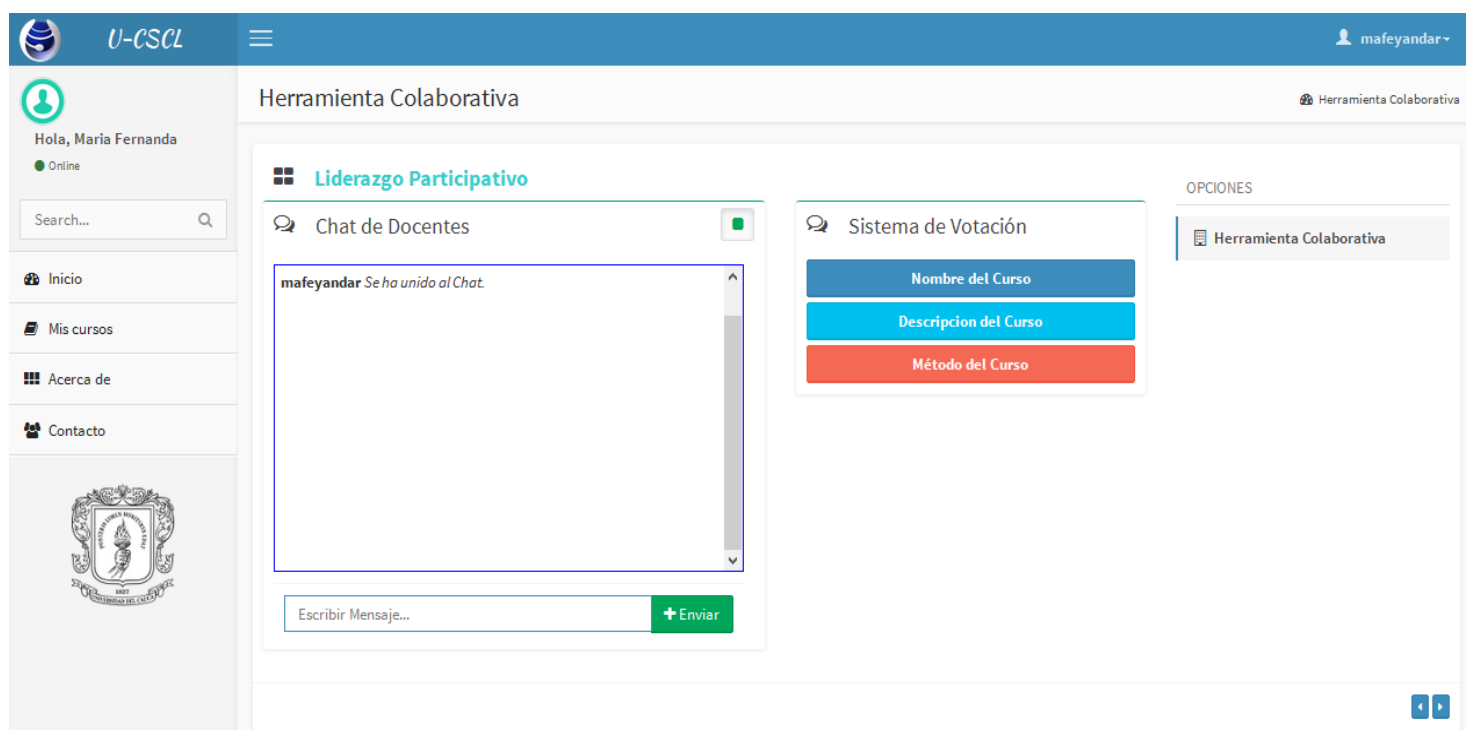

Figura 11: Herramienta colaborativa para docentes y sistema de votación Fuente:(Yandar \& Moreno, 2015) 
Los docentes también pueden comunicarse de manera asíncrona, en un espacio llamado Sugerencias, y referirse acerca de los diferentes aspectos del curso (Figura 12)

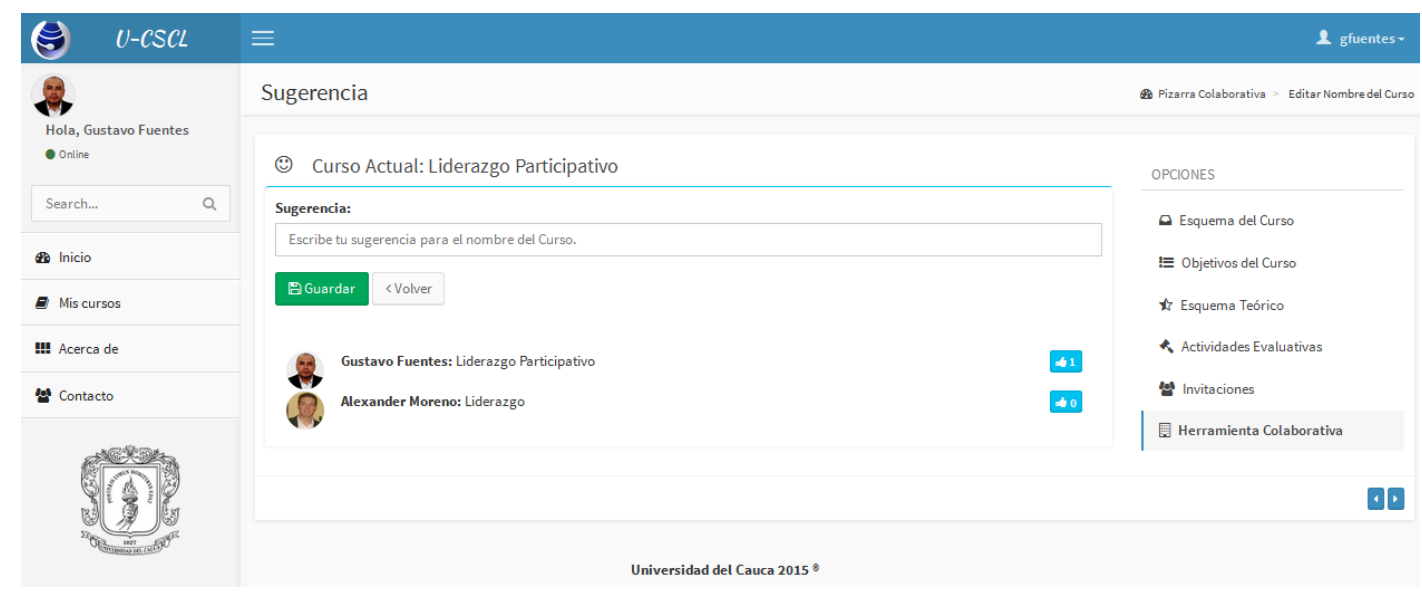

Figura 12: Comunicación asíncrona Fuente:(Yandar \& Moreno, 2015)

\subsection{Validación}

Se realizó una valoración preliminar de la herramienta en dos cursos de la Universidad del Cauca en Colombia, de cada curso se escogió aleatoriamente tres alumnos para valorar la plataforma y el modelo u-CSCL subyacente. El objetivo de esta valoración preliminar fue el de identificar aspectos de mejora de la herramienta y la facilidad de uso, mientras aún está en proceso de desarrollo, y algunos resultados iniciales en cuanto a la comprensión de la temática por parte de los estudiantes. Por tal razón no se consideró necesario contar con una muestra más amplia. En un próximo proceso (fuera del alcance de este artículo), la valoración de la plataforma se hará con grupos meta de las distintas universidades de Iberoamérica participantes y con diferentes temáticas.

Para la valoración inicial se realizaron tres sesiones:

1) La primera sesión se realizó con tres docentes y tuvo una duración de una hora, aquí se hizo una introducción de la actividad colaborativa a desarrollar, además, de explicar el funcionamiento de la herramienta.

2) La segunda sesión fue realizada con tres alumnos del programa de turismo, se explicó el funcionamiento de la herramienta y las actividades colaborativas que la componen. Para la prueba se desarrolló un curso corto sobre "liderazgo participativo" utilizando el proceso pedagógico U-CSCL y la herramienta U-CSCL.Los alumnos realizaron las actividades de forma ubicua y colaborativa varios días después.

3) La tercera sesión fue una charla sobre "Liderazgo Participativo", a tres estudiantes del programa de Física, con una duración de veinte minutos, donde se impartieron los mismos conceptos que en la clase virtual. 
Los docentes tuvieron la oportunidad de interactuar con la plataforma y valorarla en los aspectos de interactividad, apertura, usabilidad y complejidad, donde se entiende como interactividad la medida en que se propician espacios de comunicación y desarrollo entre los individuos que interactúan en la plataforma y la apertura se define como la facilidad que tiene el sistema para adaptarse a situaciones, entornos, y dispositivos, permitiendo la intervención de los usuarios desde distintas situaciones tecnológicas. Estos dos elementos fueron evaluados por cada docente con una escala Likert de 1 a 5, y los resultados se obtienen al calcular el promedio de los valores obtenidos.

Además la complejidad indica el grado de diversidad de elementos que componen una situación, los cuales se encuentran entrelazados y/o interconectados. La fórmula que se ha definido para hacer el cálculo de la complejidad percibida está dada por:

$$
c=1-\frac{\sum_{i=1}^{n} \frac{R E_{i}}{R D_{i}}}{n}
$$

Donde c es la complejidad, 1 es el valor más alto que puede tomar la complejidad; REi es el resultado evaluado en el cuestionario para la pregunta i, el cual puede tomar valores de 1 a 5; RDi es el resultado deseado en el cuestionario a la pregunta i, cuyo resultado esperado es 5 , y n es el número de preguntas evaluadas en el cuestionario (Ramírez \& Bolaños, 2013)

Por su parte, la usabilidad indica el grado en que un producto puede ser usado por determinados usuarios para lograr sus propósitos con eficacia, eficiencia y satisfacción en un contexto de uso específico. La fórmula que se definió para hacer el cálculo de la usabilidad percibida es:

$$
u s=\frac{\sum_{i=1}^{n} \frac{R E_{i}}{R D_{i}}}{n}
$$

Donde us es la usabilidad, REi es el resultado evaluado para la pregunta i, el cual puede tomar valores de 1 a 5, RDi es el resultado deseado en el cuestionario a la pregunta $\mathrm{i}$, cuyo resultado esperado es $5, \mathrm{n}$ es el número de ítems evaluados en el cuestionario (Ramírez \& Bolaños, 2013).

Las Tablas 4 y 5 muestran los resultados obtenidos para los aspectos de apertura e interacción. Como puede verse, la experiencia en general fue positiva y los docentes valoraron el potencial de la plataforma en el proceso de construcción conjunta de conocimiento. Los indicadores a evaluar del elemento Apertura están altos (ya que sobrepasan el 4 como mediana en la calificación que otorgaron los docentes), excepto en el indicador AI2 donde se refleja una pequeña dispersión, esto indica que en el trabajo síncrono, el sistema contó con un menor grado de satisfacción, con una mediana de 3 y una dispersión de más de un punto 1.33, lo cual indica que las opiniones no son unánimes. (Yandar \& Moreno, 2015). 
Tabla 4. Resultados evaluación de la herramienta U-CSCL, elemento Apertura

\begin{tabular}{|c|l|c|c|}
\hline \multicolumn{4}{|c|}{ Evaluación realizada a la herramienta colaborativa U-CSCL } \\
\hline \multicolumn{4}{|c|}{ Dimensión: tecnológica } \\
\hline Actor y evaluador: Experto \\
\hline Indicador o elemento: Apertura \\
\hline $\begin{array}{l}\text { Identificador } \\
\text { del indicador }\end{array}$ & \multicolumn{1}{|c|}{ Indicador a evaluar } & Mediana & Dispersión \\
\hline AI 1 & $\begin{array}{l}\text { El trabajo asíncrono en la } \\
\text { plataforma }\end{array}$ & 5 & 0.33 \\
\hline AI 2 & $\begin{array}{l}\text { El trabajo síncrono en el } \\
\text { sistema (comunicación en } \\
\text { tiempo real }\end{array}$ & 3 & 1.33 \\
\hline AI 3 & $\begin{array}{l}\text { Posibilidades de operación } \\
\text { con varios navegadores } \\
\text { compatibilidad con Internet, } \\
\text { Explorer, Netscape, Mozilla, } \\
\text { Firefox) }\end{array}$ & 4 & 0.33 \\
\hline AI 4 & Ubicuidad & 5 & 0 \\
\hline
\end{tabular}

Fuente:(Yandar \& Moreno, 2015).

Tabla 5: Evaluación realizada a la herramienta U-CSCL, elemento interactividad

\begin{tabular}{|c|c|c|c|}
\hline \multicolumn{4}{|c|}{ Evaluación realizada a la herramienta colaborativa U-CSCL } \\
\hline \multicolumn{4}{|c|}{ Dimensión: tecnológica } \\
\hline \multicolumn{4}{|c|}{ Actor y evaluador: Experto } \\
\hline \multicolumn{4}{|c|}{ Indicador o elemento: Interactividad } \\
\hline $\begin{array}{l}\text { Identificador } \\
\text { del indicador }\end{array}$ & Indicador a evaluar & Mediana & Dispersión \\
\hline II1 & $\begin{array}{l}\text { Servicios Interactivos de red (foro, chat, } \\
\text { videos, métodos de discusión en línea) }\end{array}$ & 5 & 0.33 \\
\hline II2 & $\begin{array}{l}\text { Gestión de actividades curriculares a } \\
\text { nivel interactivo }\end{array}$ & 5 & 0.33 \\
\hline II3 & $\begin{array}{l}\text { Herramientas tecnológicas didácticas en } \\
\text { la plataforma (Actividades colaborativas) }\end{array}$ & 4 & 0.33 \\
\hline II4 & $\begin{array}{l}\text { Adaptación del proceso pedagógico al } \\
\text { ambiente virtual }\end{array}$ & 4 & 0.33 \\
\hline II5 & $\begin{array}{l}\text { Estrategia del docente en el espacio } \\
\text { virtual }\end{array}$ & 5 & 0.33 \\
\hline
\end{tabular}

Fuente:(Yandar \& Moreno, 2015).

Los valores medios obtenidos para los criterios considerados, reflejan en general un alto grado de conformidad, con pequeñas dispersiones que indican unanimidad en las opiniones. Esto quiere decir que los docentes están de acuerdo con que la propuesta que se hace con la herramienta colaborativa U-CSCL potencia la interacción entre los participantes.

Además se realizó un test de usabilidad de la herramienta arrojando los siguientes resultados: 
Tabla 6: Evaluación realizada a la herramienta U-CSCL, elemento usabilidad

\begin{tabular}{|c|c|c|c|c|}
\hline \multirow{2}{*}{\multicolumn{5}{|c|}{$\begin{array}{c}\text { Evaluación realizada a la herramienta colaborativa U-CSCL } \\
\text { Dimensión: tecnológica }\end{array}$}} \\
\hline & & & & \\
\hline \multicolumn{5}{|c|}{ Actor y evaluador: Experto } \\
\hline \multicolumn{5}{|c|}{ Indicador o elemento: Usabilidad } \\
\hline Identificador & \multicolumn{4}{|c|}{ Indicador a evaluar } \\
\hline UI 1 & \multicolumn{4}{|c|}{ Eficacia de la participación en el espacio virtual. } \\
\hline UI 2 & \multicolumn{4}{|c|}{ Sencillez de uso del espacio virtual de la plataforma. } \\
\hline UI 3 & \multicolumn{4}{|c|}{$\begin{array}{l}\text { Facilidad de uso de las herramientas tecnológicas en la } \\
\text { plataforma. }\end{array}$} \\
\hline UI4 & \multicolumn{4}{|c|}{ Facilidad en la navegación. } \\
\hline UI 5 & \multicolumn{4}{|c|}{ Usabilidad del prototipo. } \\
\hline UI 6 & \multicolumn{4}{|c|}{ El proceso resultante es el esperado. } \\
\hline UI 7 & \multicolumn{4}{|c|}{ Facilidad y amigabilidad de la interfaz del espacio virtual. } \\
\hline \multicolumn{5}{|c|}{ Métrica utilizando fórmula para nivel de usabilidad } \\
\hline \multicolumn{5}{|c|}{$u s=\frac{\sum_{i=1}^{n} \frac{R E_{i}}{R D_{i}}}{u_{i}}$} \\
\hline \multirow{2}{*}{$\begin{array}{l}\text { Nivel de } \\
\text { usabilidad }\end{array}$} & Docente 1 & Docente 2 & Docente 3 & Promedio \\
\hline & 0.86 & 0.86 & 0.95 & 0.89 \\
\hline
\end{tabular}

Fuente:(Yandar \& Moreno, 2015).

Como se puede observar en la Tabla 6 y cotejando la fórmula que se ha definido para evaluar la Usabilidad, se puede deducir que hay un alto grado de satisfacción reflejado por los docentes al momento de responder las preguntas, considerando entonces que la herramienta colaborativa U-CSCL cumple con una alta usabilidad, es decir es una plataforma eficaz, fácil y amigable al usuario.

Para valorar la complejidad y cotejando la fórmula definida anteriormente, en la cual entre más cercano a 0 sea el valor, el grado de complejidad es menor, se muestran los resultados en la Tabla 7, puede verse que la complejidad promedio para los tres docentes que realizaron la evaluación resulta baja (0.15). Esto sugiere que la plataforma resulta sencilla de utilizar.

Tabla 7: Evaluación realizada a la herramienta U-CSCL, elemento complejidad

\begin{tabular}{|l|l|}
\hline \multicolumn{2}{|c|}{ Evaluación realizada a la herramienta colaborativa U-CSCL } \\
\hline \multicolumn{1}{|c|}{ Dimensión: tecnológica } \\
\hline Actor y evaluador: Experto \\
\hline \multicolumn{2}{|c|}{ Indicador o elemento: Complejidad } \\
\hline $\begin{array}{l}\text { Identificado } \\
\text { r }\end{array}$ & \begin{tabular}{l} 
Indicador a evaluar \\
\hline CI1
\end{tabular} \\
\hline
\end{tabular}

Modelo Colaborativo y Ubicuo para apoyar los procesos de enseñanza-aprendizaje a nivel Iberoamericano. Mayela Coto, César Collazos y Sonia Mora. 


\begin{tabular}{|c|c|c|c|c|}
\hline CI2 & \multicolumn{4}{|c|}{$\begin{array}{l}\text { Comprensión de los campos en los diferentes } \\
\text { formularios que hay que diligenciar }\end{array}$} \\
\hline $\mathrm{CI} 3$ & \multicolumn{4}{|c|}{$\begin{array}{l}\text { Comprensión de las actividades pedagógicas y } \\
\text { colaborativas, que existen en la plataforma }\end{array}$} \\
\hline \multicolumn{5}{|c|}{$c=1-\frac{\sum_{i=1}^{n} \frac{R E_{i}}{R D_{i}}}{n}$} \\
\hline $\begin{array}{l}\text { Nivel de } \\
\text { complejidad }\end{array}$ & $\frac{\text { Docente 1 }}{0.13}$ & $\begin{array}{c}\text { Docente 2 } \\
0.13 \\
\end{array}$ & \begin{tabular}{|c|} 
Docente 3 \\
0.2 \\
\end{tabular} & $\begin{array}{c}\text { Promedio } \\
0.15 \\
\end{array}$ \\
\hline
\end{tabular}

Adicionalmente, los docentes manifestaron estar de acuerdo en que la propuesta permite tener a su disposición un entorno colaborativo de apoyo a la generación del esquema del curso, que esta es ordenada y guía al docente durante el proceso. Además consideraron que la manera de expresar las actividades colaborativas para los estudiantes, es una manera entretenida de generar conocimiento, ya que emplea recursos pedagógicos innovadores que aumentan la disposición hacia el estudio. También se puede observar en el indicador de usabilidad que los docentes están de acuerdo en utilizar la herramienta U-CSCL, como entorno educativo de enseñanza-aprendizaje ya que les proporciona un entorno cómodo e intuitivo para trabajar.

Un objetivo muy importante es evaluar el grado de aprendizaje de los estudiantes, usando la herramienta, para esto se realizó una prueba corta a los dos grupos de estudiantes (los que recibieron la clase tradicional y los que interactuaron con los contenidos y las actividades colaborativas en la plataforma). Los dos métodos tuvieron el mismo docente y el mismo examen, los promedios se puede observar en la Tabla 8. Los valores promedios obtenidos para las dos evaluaciones, reflejan que el grado de aprendizaje individual es levemente mayor utilizando el "proceso para apoyar ambientes de enseñanza-aprendizaje a través de entornos ubicuos y colaborativos: U-CSCL".

Claramente este es un aspecto que debe evaluarse más y en distintas temáticas, pero de alguna manera refleja lo que se ha venido apuntando en la literatura con respecto a los procesos de aprendizaje colaborativo, en los que diversos autores mencionan, que trabajar de forma colaborativa incentiva el aprendizaje, y favorece la interdependencia positiva, ya que al realizar las actividades colaborativas en forma exitosa los miembros del grupo deben ayudarse mutuamente, asumiendo cada uno la responsabilidad individual de contribuir al aprendizaje de los demás. Además los estudiantes deben interactuar unos con otros para resolver la actividad eficientemente y en esta interacción desarrollan una serie de competencias académicas e interpersonales muy importantes para el desarrollo profesional. 
Tabla 8: Promedio obtenido por los estudiantes en la prueba

\begin{tabular}{|l|l|}
\hline $\begin{array}{l}\text { Promedio de exámenes, estudiantes evaluados aplicando el "proceso } \\
\text { para apoyar ambientes de enseñanza-aprendizaje a través de } \\
\text { entornos ubicuos y colaborativos: U-CSCL" }\end{array}$ & 4,67 \\
\hline $\begin{array}{l}\text { Promedio de exámenes para estudiantes evaluados aplicando el } \\
\text { método tradicional de cátedra. }\end{array}$ & 4,33 \\
\hline
\end{tabular}

Fuente:(Yandar \& Moreno, 2015)

\section{Conclusiones y trabajo futuro}

La innovación en la educación busca cambiar y mejorar la experiencia del aprendizaje, tanto en los alumnos como en los docentes, intentado fomentar en los estudiantes un aprendizaje individual y colectivo, permitiendo su intervención desde distintas situaciones temporales y tecnológicas, y donde el conocimiento se produzca en cualquier lugar y momento; y en los docentes se intenta fomentar procesos de colaboración que les permitan compartir sus conocimientos y contar con "expertos" en cualquier momento y lugar.

El modelo para apoyar ambientes de enseñanza-aprendizaje a través de entornos ubicuos y colaborativos: U-CSCL, propuesta realizada por un grupo de universidades a nivel Iberoamericano, combina las ventajas de un ambiente de aprendizaje colaborativo con los beneficios de la computación ubicua y la flexibilidad de las nuevas tecnologías digitales. El modelo U-CSCL es el conjunto de definiciones, roles, metodologías, actividades colaborativas, pasos, procedimientos y tecnología, destinados a regular la estructura académica y facilitar la enseñanza y el aprendizaje de manera colaborativa. Está constituido por un proceso pedagógico y tecnológico, que permite impulsar actividades colaborativas, para la transferencia del conocimiento. También se destaca la elaboración de una plataforma colaborativa, ubicua y enfocada a la educación, para el apoyo del proceso U-CSCL, que ofrece la posibilidad de crear y desarrollar colaborativamente distintos cursos virtuales, aplicables de manera colaborativa y ubicua.

La versión actual de la herramienta permitió a los docentes crear, de forma colaborativa y ubicua, la estructura de un curso, logrando integrar a un conjunto de personas y sus opiniones, en busca de un objetivo en común, donde cada uno aportó su punto de vista a partir de sus conocimientos y experiencia. Además, permitió a los estudiantes aprender sobre la temática de "Liderazgo participativo" de forma ubicua y colaborativa, participar en las actividades colaborativas propuestas en el proceso, propiciando así el desarrollo de conocimiento conjunto, y permitiendo un grado de aprendizaje superior que el aprendizaje obtenido por el método de clase tipo cátedra, evaluado mediante una prueba corta. Es claro que se debe seguir validando el entorno, para obtener mayores resultados y más precisos. Esto mediante la generación de contenidos digitales en otras áreas del conocimiento. Si bien el número de participantes es poco para obtener datos significativamente interesantes, se trata de una valoración muy preliminar, en la cual se pueden intuir resultados interesantes. 
Como trabajo futuro, aún queda mucho por hacer, se debe trabajar en la construcción de nuevos y diversos cursos, también se deben hacer más evaluaciones de la plataforma y darle seguimiento a la misma. Adicionalmente, se plantea a futuro incluir técnicas de gamificación en la elaboración de las actividades colaborativas de los estudiantes.

\section{Agradecimientos}

Este artículo es desarrollado como parte del proyecto "RED u-CSCL: Red Iberoamericana de apoyo a los procesos de enseñanza-aprendizaje de competencias profesionales a través de entornos ubicuos y colaborativos, código CYTED 513RT0481" y del proyecto de la Universidad Nacional, "Desarrollo e Implementación de un Modelo Pedagógico de Apoyo a los Procesos de Enseñanza-Aprendizaje de Competencias Profesionales a través de entornos ubicuos y colaborativos en el marco de la red U-CSCL", código 0406.

Presentación del artículo: 25 de noviembre de 2015 Fecha de aprobación: 23 de diciembre de 2015

Fecha de publicación: 30 de enero de 2016

Coto, M., Collazos, C., Mora, S. (2016). Modelo Colaborativo y Ubicuo para apoyar los procesos de enseñanza-aprendizaje a nivel Iberoamericano. RED. Revista de Educación a Distancia. 48(10). Consultado el (dd/mm/aaaa) en http://www.um.es/ead/red/48/nolasco.pdf

\section{Bibliografía}

Acosta, C. (2010). Diseño e implementación de una herramienta de Representación del conocimiento para apoyar la Gestión de requisitos en un proceso de desarrollo de software. Universidad de Chile.

Badia, A. (2005). Aprender a colaborar con Internet en el aula. En C. Monereo (Ed.), Internet y competencias básicas (pp. 93-116). Barcelona: Editorial Graó.

Barkley, E., Cross, K. P., \& Major, C. H. (2005). Collaborative learning techniques: A Handbook for college faculty. San Francisco: Jossey-Bass.

Briggs, R., et al. (2006). Defining key concepts for collaboration engineering. Presentado en Americas Conference on Information Systems.

Briggs, R., Vreede, G., \& Nunamaker, J. (2003). Collaboration engineering with ThinkLets to pursue sustained success with group support systems. Journal of Management Information Systems, 19, 31-64.

Cabero, J., \& Llorente, M. (2008). La alfabetización digital de los alumnos. Competencias digitales para el siglo XXI. Revista Portuguesa de Pedagogía, 42(2), 7-28. 
Calzadilla, M. E. (2002). Aprendizaje colaborativo y tecnologías de la información y la comunicación. Revista Iberoamericana de Educación, 1(10).

Collazos, C., et al. (2007). Evaluating Collaborative Learning Processes using Systembased Measurement. Educational Technology \& Society, 10(3), 257-274.

Collazos, C., \& Mendoza, J. (2006). Como aprovechar el aprendizaje colaborativo en el aula. Revista Educación y Educadores, 9(2), 61-76.

Collazos, C., et al. (2013). Propuesta metodológica de apoyo a los procesos de enseñanza-aprendizaje a través de entornos ubicuos y colaborativos: u-CSCL. Presentado en VIII Congreso Colombiano de Computación - 8CCC.

Collazos, C., \& Solano, A. (2014). Creación de Objetos de Aprendizaje desde un Enfoque Colaborativo. En Objetos de Aprendizaje de Contenidos Abiertos Accesibles: Del Diseño a la Reutilización (Vols. 1-1era, pp. 49-68). Iniciativa Latinoamericana de Libros de Texto Abiertos (LATIn).

Dillenbourg, P. (1999). Collaborative learning: cognitive and computational approaches (1st ed.). Amsterdam/New York: Pergamon. Recuperado de http://www.loc.gov/catdir/enhancements/fy0601/98038305-d.html

Fjermestad, J., \& Hiltz, S. (2001). Group Support Systems: A descriptive evaluation of case and field studies. Journal of Management Information Systems, 17(3), 112-157.

Gros, B., \& Silva, J. (2005). La formación del profesorado como docente en los espacios virtuales de aprendizaje. Revista Iberoamericana de Educación, 36.

Johnson, D., \& Johnson, R. (1975). Learning together and alone. Cooperation, competition and individualization. Englewood Cliffs, New Jersey.: Prentice Hall Inc.

Johnson, D., Johnson, R., \& Holubec, E. (1992). Advanced cooperative learning. Edina, $\mathrm{MN}$ : Interaction Books.

Kirschner, F., Paas, F., \& Kirschner, P. A. (2009). Individual and group-based learning from complex cognitive tasks: Effects on retention and transfer efficiency. Computers in Human Behavior, 25, 306-314.

Kolfschoten, G., et al. (2004). ThinkLets as building blocks for collaboration processes: a further conceptualization. Groupware: Design, Implementation and Use, 137-152.

Kolfschoten, G., Briggs, R., \& Vreede, G. (2006). Definitions in Collaboration Engineering. Presentado en Proceedings of the 39 Hawaii International Conference on System Sciences, Delft University of Technology, University of Arizona.

Kolfschoten, G., \& Vreede, G. (2007). The Collaboration Engineering Approach for Designing Collaboration Processes. Presentado en International Conference on Groupware: Design, Implementation and Use. 
Kolfschoten, G., \& Vreede, G. (2009). A Design Approach for Collaboration Processes: A Multimethod Design Science Study in Collaboration Engineering. Journal of Management Information Systems, 6(1), 225-256.

Kolfschoten, G., Vreede, G., \& Briggs, R. (2010). Collaboration 'Engineerability'. Group Decision and Negotiation, 19(3), 301-321.

Koschman, T., Kelson, A., Feltovich, P., \& Barrows, H. (1996). Computer-supported problem-based learning: A principled approach to the use of computers in collaborative learning. CSCL: Theory and practice of an emerging paradigm, 83-124.

Lipponen, L. (2002). Exploring foundations for computer-supported collaborative learning (pp. 72-81). International Society of the Learning Sciences.

Méndez, Y., et al. (2008). Thinklets: Un Artefacto Útil para el Diseño de Métodos de Evaluación de la Usabilidad Colaborativa. Revista Avances en Sistemas e Informática, 5(2), 147-154.

Muhlenbrock, M. (1999). A system for Analyzing Collaborative problem solving. Recuperado de http://citeseer.nj.nec.com/410548.html

Ramírez, D., \& Bolaños, J. (2013). Guía para el diseño de actividades de aprendizaje colaborativo asistida por computador. Popayán, Colombia: Universidad del Cauca. Facultad de Ingeniería Electrónica y Telecomunicaciones. Departamento de Sistemas.

Roschelle, J., et al. (2000). Changing how and what children learn in school with computer-based technologies. Future Child, 10(2), 76-101.

Rubia, B., Jorri, I., \& Anguita, R. (2009). Aprendizaje colaborativo y TIC. En J. D. P. Pons (Ed.), Tecnología Educativa. La formación del profesorado en la era de Internet. Málaga: Aljibe.

Salinas, M. I. (2011). Entornos virtuales de aprendizaje en la escuela: tipos, modelo didáctico y rol del docente. Buenos Aires: PROSED-UCA.

Santanen, E., \& Vreede, G. (2004). Creative Approaches To Measuring Creativity: Comparing The Effectiveness Of Four Divergence Thinklets. Presentado en 37th Hawaiian Internal Conference On System Sciences, Los Alamitos, IEEE Computer Society Press.

Scagnoli, N., \& Stephens, M. (2005). Collaborative learning strategies in online education. Presentado en Online Conference for Teaching and Learning (IOC2005), Illinois.

Solano, A., \& Collazos, C. (2013). Modelo para el diseño de actividades colaborativas desde un enfoque práctico. Revista Universitaria RUTIC, 1(2). Recuperado de http://ciclope.unicauca.edu.co/rutic/index.php/rutic/article/view/169 
Stahl, G. (2004). Building collaborative knowing: Elements of a social theory of CSCL. En J.-W. Strijbos, P. A. Kirschner, \& R. Martens (Eds.), What we know about CSCL: And implementing it in higher education (pp. 53-86). Boston, Mass.: Kluwer Academic Publishers.

Tatar, D., et al. (2003). Handhelds: Go to Scholl: Lessons Learned. Washington DC.: IEEE Computer Society.

Vreede, G., Davison, R., \& Briggs, R. (2003). How A Silver Bullet May Lose Its Shine - Learning From Failures With Group Support Systems. Communications of the ACM. 46(8), 96-101.

Yahya, S., Ahmad, E. A., \& Jalil, K. A. (2010). The definition and characteristics of ubiquitous learning: A discussion. International Journal of Education and Development using Information and Communication Technology (IJEDICT), 6(1), 117-127.

Yandar, M. F., \& Moreno, A. (2015). Definición de un proceso para apoyar ambientes de enseñanza-aprendizaje a través de entornos ubicuos y colaborativos: U-CSCL (Trabajo de Pregrado). Colombia, Popayán: Universidad del Cauca. Facultad de Ingeniería Electrónica y Telecomunicaciones. Departamento de Sistemas. 